\title{
Physical Layer Performance Modeling of Modern Multicarrier Modulation Techniques
}

This paper was downloaded from TechRxiv (https://www.techrxiv.org).

\section{LICENSE}

CC BY 4.0

SUBMISSION DATE / POSTED DATE

02-09-2021 / 10-09-2021

\section{CITATION}

Anwar, Waqar; Kumar, Atul; Franchi, Norman; Fettweis, Gerhard (2021): Physical Layer Performance Modeling of Modern Multicarrier Modulation Techniques. TechRxiv. Preprint. https://doi.org/10.36227/techrxiv.16563636.v1

DOI

10.36227/techrxiv.16563636.v1 


\title{
Physical Layer Performance Modeling of Modern Multicarrier Modulation Techniques
}

\author{
Waqar Anwar, Member, IEEE, Atul Kumar, Member, IEEE, Norman Franchi, Member, IEEE, \\ and Gerhard Fettweis, Fellow, IEEE.
}

\begin{abstract}
The fifth-generation (5G) and beyond communications are facing the challenge to meet the diverse requirements of modern use cases. Multicarrier modulation techniques are the key component of physical layer (PHY) design, which has the potential to improve the efficiency and reliability of current communications and hence to address the challenge. In stateof-the-art wireless technologies (i.e., NR and IEEE 802.11ax) orthogonal frequency division multiplexing (OFDM) is employed at PHY, although it has many disadvantages, i.e., peak-toaverage power ratio (PAPR), out-of-band emission (OOBE), and sensitivity to carrier frequency offset (CFO). To overcome OFDM drawbacks several multicarrier modulation techniques are being considered, such as discrete Fourier transform-spreadOFDM (DFT-s-OFDM), generalized frequency division multiplexing (GFDM), and orthogonal time frequency space (OTFS). In this paper, we develop the physical layer abstraction (PLA) of these multicarrier techniques to evaluate and compare their performance in various use cases and scenarios. The PLA is commonly used in system-level simulators to avoid timeconsuming PHY simulations. The validation results show that performance can be accurately estimated through PLA at least thousands times faster. Besides, the developed PLA is utilized to compare and evaluate the performance of multicarrier techniques in different fading conditions.
\end{abstract}

Index Terms-Physical layer abstraction, system-level simulation, link adaptation, multicarrier modulation techniques, eEESM, EESM, RBIR, OFDM, DFT-s-OFDM, GFDM, OTFS

\section{INTRODUCTION}

The physical layer (PHY) is continuously evolving with each new generation of a mobile communications standard. In current cellular transmission schemes, such as Long-Term Evolution (LTE) and fifth generation new radio (5G NR), orthogonal frequency division multiplexing (OFDM) is employed. Although OFDM is robust against the frequencyselectivity of the channel, it has some disadvantages such

This work has been submitted to the IEEE for possible publication. Copyright may be transferred without notice, after which this version may no longer be accessible.

This work was supported in parts by the project "Industrial Radio Lab Germany" under contract 16KIS1010K, funded by the Federal Ministry of Education and Research (BMBF), Germany. The authors alone are responsible for the content of the paper.

W. Anwar, A. Kummar, and G. Fettweis are affiliated with Vodafone Chair Mobile Communications Systems, Department of Electrical Engineering, Technische Universität Dresden, Germany (E-mail: \{waqar.anwar, atul.kumar, norman.franchi, gerhard.fettweis $\}$ @tu-dresden.de).

N. Franchi is affiliated with Institute for Electrical Smart City Systems, Friedrich-Alexander-Universität Erlangen-Nürnberg, Germany (E-mail: norman.franchi@fau.de).

This paper in part was presented at the IEEE Global Communication Conference (GLOBECOM), Waikoloa, Hawaii, USA, Dec. 2019 [1] as out-of-band emission (OOBE), peak-to-average power ratio (PAPR), and sensitivity to carrier frequency offset (CFO). To address the issue of high PAPR discrete Fourier transform (DFT)-spread-OFDM (DFT-s-OFDM) is defined for uplink transmissions in the LTE/NR. However, both OFDM and DFTs-OFDM become inter-carrier interference (ICI) limited in high mobility scenarios (due to Doppler impairments) such as enhanced vehicle-to-everything (eV2X) communications. Therefore, new multicarrier modulation schemes are under investigation for beyond $5 \mathrm{G}$ communications. Another multicarrier technique mainly proposed to reduce the OOBE is the "generalized frequency division multiplexing (GFDM)". It implies circular filtering at the subcarrier level to suppress OOBE. However, the main problems of GFDM are the non-orthogonality generated by its prototype filters and sensitivity to CFO. In order to overcome the above challenges a new multicarrier modulation "orthogonal time frequency space (OTFS)" is being proposed [2]. In OTFS symbols are localized in the delay-Doppler domain and the cyclic prefix (CP) protects orthogonality in both domains. Consequently, Doppler impairments can be mitigated and sensitivity to CFO could be reduced [3]. Among multicarrier techniques under consideration, the most suitable for $\mathrm{eV} 2 \mathrm{X}$ seems to be OTFS.

In order to evaluate upcoming technologies (having different multicarrier techniques), under various fading conditions, system-level simulations are essential. As a large number of nodes are involved in system-level evaluations; therefore, PHY simulations of each individual node becomes infeasible due to the huge processing required at PHY. To speed up systemlevel simulations physical layer abstraction (PLA) is generally used in system-level simulators. Especially, it models the PHY performance, e.g., packet error rate (PER) and throughput, exclusively as a function of the received signal-to-interferenceplus-noise ratio (SINR). Nevertheless, the received symbols in wideband multicarrier communications under doubly selective (time-frequency) fading could have variable channel gain, consequently, the received SINR of these symbols might be different. In order to obtain a single link quality indicator (LQI), the instantaneous SINRs of received symbols are mapped to an effective SINR using PLA. Now, the effective SINR could be used to predict PHY performance in the case of system-level simulations or to select optimal modulation and coding scheme (MCS) for link adaptation. In addition, various multicarrier techniques could perform different even under similar fading condition, therefore multicarrier modulation specific PLA modeling is required. 


\section{A. Related Work}

In literature, exponential effective SINR mapping (EESM)) [4], [5] and received bit information rate (RBIR) [6], [7] are the most commonly used effective SINR mapping algorithms. These algorithms are used to compute effective SINR of a received packet when symbols have variable SINR due to fading. Several studies compare and evaluate the performance of these algorithms in system-level simulations and in link adaptation [5]-[10]. Results show that SINR mapping schemes can precisely predict $\mathrm{PHY}$ performance without passing actual data through PHY chain. However, the prediction accuracy mainly depends on the channel profile and used MCS, as a result, MCS and channel specific optimization is required. Later in [11], another effective SINR mapping algorithm named enhanced exponential effective SINR mapping (eEESM) is proposed which outperforms existing state-of-the-art (i.e., EESM and RBIR) algorithms. In this later study, authors abstract the performance of different vehicle-to-everything (V2X) technologies and compare their performance in an Urban line-of-sight (LOS) V2X channel model. Further in [12], the eEESM is applied to multi-connectivity networks with link combining schemes. Nevertheless, the main focus of so far available studies is to abstract the performance of OFDM based systems in frequency selective fading (FSF) channel.

In the context of future wireless technologies, various multicarrier techniques can be used to meet application-specific requirements. Moreover, in high mobility use cases, the channel is not only frequency selective but also time selective. As discussed earlier, in the case of eV2X doubly selective fading (DSF) will play a key role where new multicarrier techniques are expected to perform better as analyzed in [13]. These multicarrier techniques could have different symbol SINRs even under similar fading conditions. As a result, the modeling of effective SINR of each multicarrier technique is required by taking into account the ICI effect due to Doppler and time and frequency selectivity of the channel. Therefore, in this paper, we model these shortcomings by developing an enhanced PLA which accurately abstracts the characteristics of different multicarrier techniques under various fading conditions. We validate the performance of developed PLA in each case by comparing the estimation accuracy against PHY simulations. Lastly, we utilize developed PLA to evaluate the performance among multicarrier techniques under various fading conditions. To the best of our knowledge, we are the first to propose a multicarrier-specific approach to abstract the PHY performance in both time and frequency selective channels.

\section{B. Contribution}

In this paper, we develop an enhanced PLA for modern multicarrier techniques (i.e., OFDM, DFT-s-OFDM, GFDM, and OTFS) to predict their performance under different fading conditions. To abstract the performance of multicarrier techniques, we derive the received SINR expressions for multicarrier techniques by taking into account the impact of ICI. Then the multiple received SINRs in a packet due to fading are mapped to effective SINR using eEESM. This takes into account the impact of channel selectivity on the received symbols transmitted through these multicarrier techniques. To achieve optimum mapping accuracy of eEESM in different fading conditions, the optimization of a fitting parameter is required. This optimization usually depends on multicarrier techniques, MCS order, and fading conditions. In order to reduce optimization complexity, we model optimization parameter as a function of the mapping SINRs which makes it independent of fading conditions. Therefore, the resultant effective SINR provides a more realistic performance evaluation in system-level simulations and enables reliable link adaptation. Furthermore, to verify the accuracy of PLA, PHY simulators of multicarrier techniques are implemented in MATLAB. The performance of proposed PLA modeling is quantified in terms of mean squared error (MSE) and compared with full PHY simulations. Subsequently, a comparison between PHY simulations and PLA is provided in terms of simulation time. In the end, applications of PLA are showcased by comparing the performance of multicarrier techniques under various fading conditions, e.g., FSF and DSF.

\section{Organization}

The paper is organized as follows. In Section II, an overview of multicarrier modulation techniques is provided and their pros and cons are discussed. In Section III, the ICI and its impact on communication performance is discussed. Afterward, the system model of multicarrier modulation techniques is described and SINR expressions are derived in Section IV. Further, in Section V, the eEESM based effective SINR mapping is explained, and its optimization against different fading conditions is discussed. In addition, the end-to-end multicarrier performance modeling using PLA under various fading conditions is presented. Subsequently, the performance of multicarrier PLA is evaluated against PHY simulations in Section VI, and error is quantified in terms of root-meansquare error (RMSE). Later in Section VII, the performance of multicarrier techniques is compared under various fading conditions using PLA. Finally, the main findings are discussed in Section VIII.

\section{Overview of MulticARrier TeChNiques}

Multicarrier modulations divide a broadband channel into closely spaced subcarriers for data transmissions. It offers multiple advantages over single carrier transmissions, which include resilience to narrowband fading and multipath effects. Furthermore, in the FSF channel, each subcarrier experiences flat fading which can be equalized using single tab equalization. Therefore, multicarrier techniques are widely used in the current state-of-the-art wireless standards. The timefrequency domain representation of transmitted symbols using various multicarrier techniques is shown in Fig. 1. Further explanation of their modulation principle and the pros and cons are discussed below.

\section{A. Orthogonal Frequency Division Multiplex (OFDM)}

OFDM divides a wideband channel into $N$ parallel narrowband sub-channels such that each sub-channel can experience frequency flat behavior. The serial stream of transmitted 


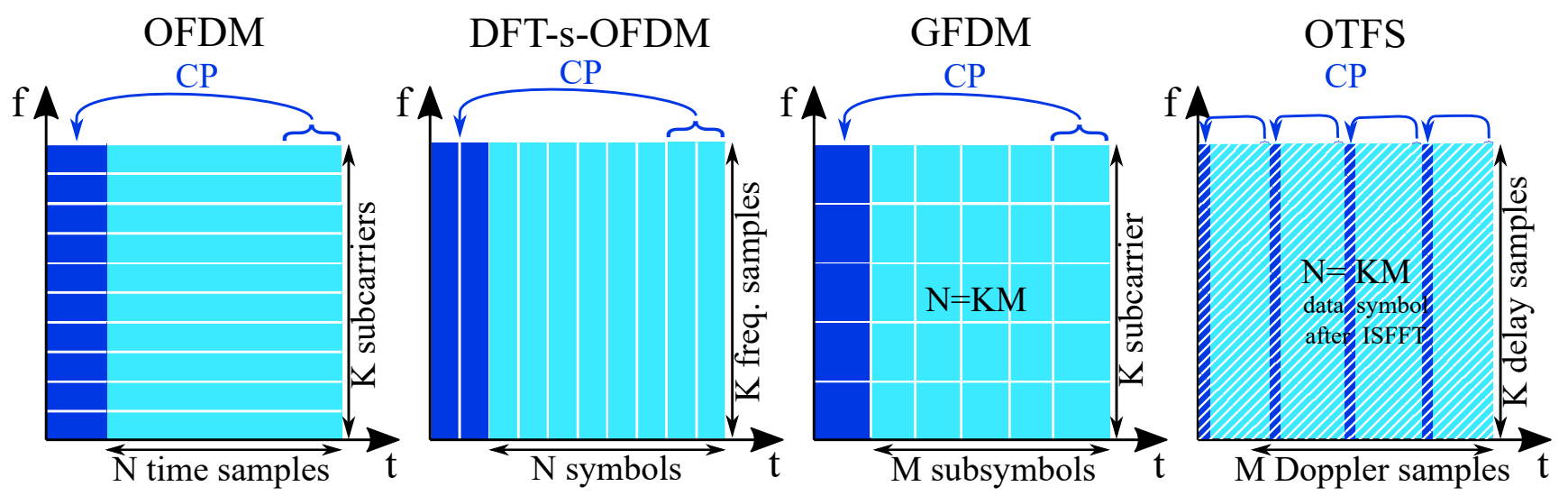

Fig. 1: Time-frequency representation of multicarrier modulation techniques

symbols is converted into $N$ parallel streams, which are then mapped to $N$ orthogonal subcarriers. The advantage of dividing the channel into multiple sub-channels is that a single tap equalization per subcarrier is possible that reduces the complexity and increases the spectral efficiency of the system. Furthermore, a CP is appended at the end of each OFDM symbol to mitigate inter-symbol interference (ISI) in multipath channels. It is currently being used by many wireless technologies, e.g., LTE, NR, and IEEE 802.11ac/ax. Its main advantages are easy equalization and low complexity [14], whereas disadvantages include high PAPR, OOBE, and sensitivity to CFO. In order to achieve better performance, many other multicarrier techniques have been proposed [2], [14].

\section{B. DFT-Spread-OFDM (DFT-s-OFDM)}

The DFT-s-OFDM, also known as single carrier frequency division multiplexing (SC-FDM), is similar to OFDM with an additional DFT precoding that helps to reduce PAPR and improve the power efficiency of RF amplifiers. Therefore, it is suitable for low-budget devices and thus being used in LTE and NR for uplink transmissions. The transmitted symbols are first processed by $M$-point DFT, followed by resources mapping and $N$-point inverse DFT (IDFT) stage. It is important to mention here that $M$ must be smaller than $N$, otherwise, for $M=N$ the cascaded DFT and IDFT processing would cancel each other out. For this reason, the value of $M$ is selected such that $N$ becomes the multiple of $M$. There are two ways to map the DFT outputs to the subsequent IDFT inputs: either localized or interleaved. More specifically, the output of DFT is either mapped to consecutive or uniformly spaced inputs of the OFDM modulator. Similar to OFDM, CP is used to avoid ISI and single tab equalization can be used after IDFT stage at the receiver. Disadvantages of DFT-s-OFDM are: increased complexity (referenced to OFDM), OOBE, and sensitivity to CFO [14].

\section{Generalized Frequency Division Multiplexing (GFDM)}

The GFDM is another multicarrier modulation technique that uses a circular pulse shaping filter to reduce OOBE. The transmitted symbols are arranged into $K \times M$ blocks where
$K$ is the number of subcarriers and $M$ is the number of subsymbols [14]. Afterward, a CP is appended to each GFDM block to maintain circularity and to ensure linear equalization at the receiver. The key step in GFDM modulation is the use of a digital pulse shaping filter at the transmitter and matched filter at the receiver. For that, the transmitted data is first up-sampled and then convolved with a pulse-shaping filter, e.g., a root-raised cosine function. Subsequently, the signal is match filtered at the receiver and down-sampled. Finally, an equalization in frequency domain (e.g. zero-forcing (ZF) and minimum mean squared error (MMSE)) can be used to recover the transmitted symbols [15]. The main benefit of GFDM is that using a special set of design parameters earlier introduced multicarrier techniques, i.e., OFDM, and DFT-s-OFDM can be generated, hence named as generalized. Disadvantage is, in the presence of Doppler or CFO the orthogonality gets lost which introduces ICI similar to previous techniques. To overcome ICI, an iterative interference equalizer could be used however it significantly increases GFDM complexity [14].

\section{Orthogonal Time Frequency Space (OTFS)}

The OTFS is a newly proposed multicarrier technique that uses a different approach by interpreting channels in the delayDoppler domain contrary to traditionally used time-frequency domain. The fading channel can be characterized in the delayDoppler domain by its delay taps $\tau$ and Doppler shifts $\nu$, given as $h(\tau, \nu)$. Both channel representations are interrelated and conversion is possible using symplectic finite Fourier transform (SFFT). At the transmitter, symbols are arranged in the delay-Doppler grid and inverse SFFT (ISFFT) followed by Heisenberg transform is used to generate the time domain waveform. A Wigner Transformation and SFFT at the receiver could be used to convert symbols back into the delay-Doppler domain. Alternatively, it can also be implemented in the OFDM framework by precoding symbols using ISFFT before OFDM modulation at the transmitter and the associated SFFT after OFDM demodulation at the receiver. In OTFS, symbols span the whole bandwidth in the frequency domain and the duration of a packet in the time domain. This enables OTFS to fully exploit the inherent time-frequency diversity of the channel. Moreover, a CP added in the time domain helps 
TABLE I: Time selectivity $\left(\tau_{s}\right)$ and ICI $\left(\sigma_{\nu_{\mathrm{ICI}}}^{2}\right)$ for various combinations of Doppler shift and carrier spacing

\begin{tabular}{|c|c|c|c|c|c|c|c|c|}
\hline \multirow{2}{*}{ Doppler shift } & \multicolumn{2}{|c|}{$\begin{array}{c}15 \mathrm{kHz} \\
\left(\mathbf{L T E} / \mathrm{NR}^{0}\right)\end{array}$} & \multicolumn{2}{|c|}{$\begin{array}{c}60 \mathrm{kHz} \\
\left(\mathrm{NR}^{2}, \approx 802.11 \mathrm{ax}\right)\end{array}$} & \multicolumn{2}{|c|}{$\begin{array}{c}156.25 \mathrm{kHz} \\
(802.11 \mathrm{p} / 11 \mathrm{bd})\end{array}$} & \multicolumn{2}{|c|}{$\begin{array}{l}312.5 \mathrm{kHz} \\
(802.11 \mathrm{ac})\end{array}$} \\
\hline & $\tau_{s}$ & $\sigma_{\nu_{\mathrm{ICI}}}^{2}$ & $\tau_{s}$ & $\sigma_{\nu_{\mathrm{ICI}}}^{2}$ & $\tau_{s}$ & $\sigma_{\nu_{\mathrm{ICI}}}^{2}$ & $\tau_{s}$ & $\sigma_{\nu_{\mathrm{ICI}}}^{2}$ \\
\hline $100 \mathrm{~Hz}$ & 0.0067 & $-38.4 \mathrm{~dB}$ & 0.0017 & $-50.5 \mathrm{~dB}$ & 0.00064 & $-58.9 \mathrm{~dB}$ & 0.00032 & $-64.9 \mathrm{~dB}$ \\
\hline $500 \mathrm{~Hz}$ & 0.033 & $-24.5 \mathrm{~dB}$ & 0.0083 & $-36.5 \mathrm{~dB}$ & 0.0032 & $-44.9 \mathrm{~dB}$ & 0.0016 & $-51.5 \mathrm{~dB}$ \\
\hline $1000 \mathrm{~Hz}$ & 0.067 & $-18.4 \mathrm{~dB}$ & 0.017 & $-30.5 \mathrm{~dB}$ & 0.0064 & $-38.9 \mathrm{~dB}$ & 0.0032 & $-45.5 \mathrm{~dB}$ \\
\hline $2000 \mathrm{~Hz}$ & 0.13 & $-12.4 \mathrm{~dB}$ & 0.033 & $-24.5 \mathrm{~dB}$ & 0.013 & $-32.9 \mathrm{~dB}$ & 0.0064 & $-38.9 \mathrm{~dB}$ \\
\hline
\end{tabular}

to maintain the orthogonality in the delay-Doppler domain, hence with a suitable equalizer delay-Doppler interference can be nullified. Therefore, it is a favourable multicarrier technique for transmissions in high Doppler scenarios like eV2X communications. As it exploits time-frequency selectivity of the channel as an additional source of diversity instead of interference [16].

\section{INTER-CARRIER INTERFERENCE}

Multicarrier modulation techniques use $\mathrm{CP}$ to ensure the orthogonality of subcarriers under frequency dispersive channels. However, in $\mathrm{eV} 2 \mathrm{X}$ communications, channels are time dispersive in nature due to Doppler shift [17], [18]. The Doppler shift cause interference among subcarriers in a multicarrier system, such as OFDM, also known as ICI. The impact of time dispersion could be determined by the product of maximum Doppler shift and symbol duration (inverse of subcarrier spacing). In other words, if symbol duration is very large a smaller Doppler shift can also result in a reasonable ICI. Although, the used pulse shaping filter also plays a crucial role. In the case of OFDM, the variance of ICI at $k$-th subcarrier can be expressed (from [19]) as,

$$
\sigma_{\nu}^{2}(k)=\sum_{n=1, n \neq k}^{N}\left|P\left(\frac{n-k}{T_{\mathrm{s}}}+f_{\mathrm{D}}\right)\right|^{2},
$$

where $T_{\mathrm{s}}$ is the symbol duration, $P($.$) is the pulse shaping$ filter, $N$ is the number of subcarriers, and $f_{D}$ is the maximum Doppler shift. Many studies have investigated the impact of pulse shaping filters in reducing ICI power [19]-[22]. These studies show that the impact of ICI could be significantly reduced using advanced pulse shaping filters, e.g., raised cosine filter, improved sinc-power, and Nyquist-I. Nevertheless, the rectangular pulse shaping filter is currently being used by most state-of-the-art wireless technologies. The performance degradation due to Doppler can also be analyzed in terms of signal to interference ratio (SIR), given as

$$
\mathrm{SIR}=\frac{\left|P\left(f_{\mathrm{D}}\right)\right|^{2}}{\sum_{n=1, n \neq k}^{N}\left|P\left(\frac{n-k}{T_{\mathrm{s}}}+f_{\mathrm{D}}\right)\right|^{2}} .
$$

It can be observed from (1) that for a given pulse shaping filter, the ICI mainly depends on two factors, i.e., $f_{\mathrm{D}}$ and $T_{s}$. The ratio of these two factors can also be termed as time selectivity of the channel $\left(\tau_{s}=f_{\mathrm{D}} \cdot T_{s}\right)$. In Fig. 2, values of ICI (on the left side) and SIR (on the right side) are plotted as a function of $\tau_{s}$ for the case of rectangular pulse shaping filter.

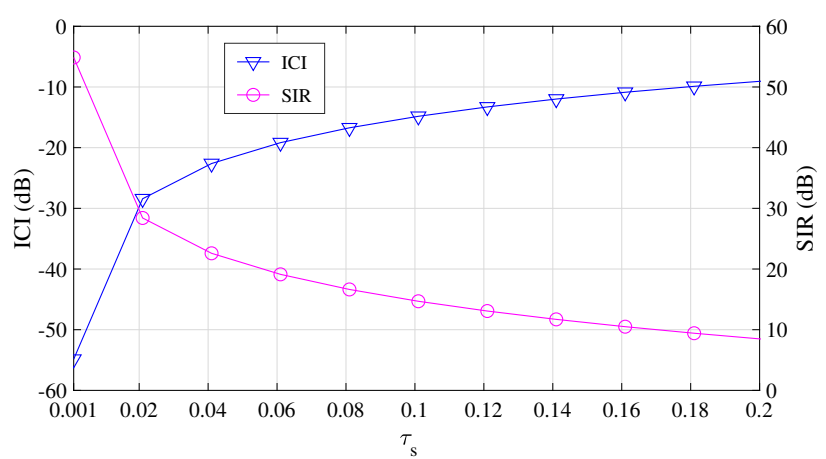

Fig. 2: ICI and SIR value for various values of $\tau_{s}$

Results show that with the increase in ratio from 0.02 to 0.2 the ICI power increases by $\approx 25 \mathrm{~dB}$, hence SIR reduces from $29 \mathrm{~dB}$ to $8 \mathrm{~dB}$. These results demonstrate that in the case of a higher $\tau_{s}$ system becomes ICI denominated and performance could not be improved by increasing the transmit power. Furthermore, the ICI variance for various combinations of carrier spacing and Doppler shift is provided in Table I. The choice of carrier spacing plays an important role to improve performance in high Doppler scenarios. For example, the LTE-V2X could become ICI dominated even at $500 \mathrm{~Hz}$, whereas using $60 \mathrm{kHz}$ carrier spacing (defined by NR with $u=2$ ) SIR can be improved by $\approx 12 \mathrm{~dB}$. Similarly, due to lower carrier spacing used by IEEE $802.11 \mathrm{p} / \mathrm{bd}, 20 \mathrm{~dB}$ higher SIR compared to LTE/ $\mathrm{NR}^{0}$ can be achieved. Although the increase in carrier spacing can reduce ICI, it negatively affects the robustness against frequency dispersion. This is due to the decrease in symbol duration and consequently the CP. Therefore, it must be considered while selecting an optimal carrier spacing. Finally, it is important to mention that the ICI can also be minimized using iterative interference cancellation schemes [23]-[26].

\section{SYSTEM MODEL}

The multicarrier modulation system model is illustrated in Fig. 3. At transmitter the data packet $\mathbf{D}$ is first encoded using LDPC codes (as defined by NR) then $M$-QAM constellations mapping is performed. The resulting symbols are further processed by multicarrier-specific modulators and a time domain signal $s(t)$ is generated. The transmitted signal is then convolved with a multipath channel denoted as $h_{t}(\tau)$ and additive white Gaussian noise (AWGN) noise is added. Subsequently, at the receiver side signal is demodulated, channel estimation and equalization are performed. Following assumptions are 


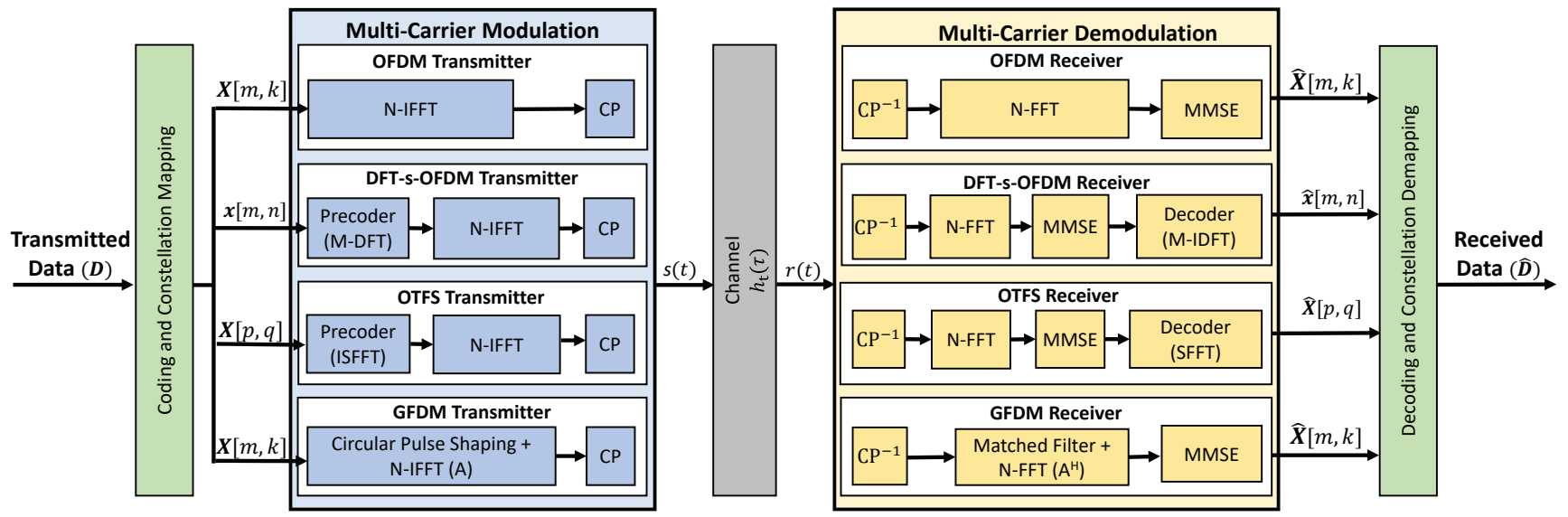

Fig. 3: Multicarrier modulation and demodulation system model

made to simplify modeling: perfect synchronization is possible in time and frequency and error-free channel estimation is available at the receiver. In the later part of this section, the step-by-step processing of each multicarrier technique is discussed and the received SINR expressions are derived.

Symbols and notations: the frequency domain matrix is denoted with a capital bold symbol, the time domain matrix is denoted by a small bold symbol, the frequency domain signal is denoted by a capital symbol, the time domain signal is denoted by a small symbol, the transpose operator is denoted by $(\cdot)^{T}$, the Hermitian operation is denoted by $(\cdot)^{H}$, and identity matrix is denoted by $\mathbf{I}$.

\section{A. OFDM}

The data packet (D) after encoding and constellation mapping could be represented by a matrix $\mathbf{X}$ of $M \times K$ dimensions, where $M$ is the number of OFDM symbols and $K$ is the number of data subcarriers. The time-duration of an OFDM symbol is $T_{s}$, where subcarrier spacing is equal to $\frac{1}{T_{s}}$. The modulated symbols $\mathbf{X}$ inside a packet could be expressed as

$$
\mathbf{X}=[\mathbf{X}(1), \mathbf{X}(2), \ldots, \mathbf{X}(M)]
$$

here

$$
\mathbf{X}(m)=[X(m, 1), X(m, 2), \ldots, X(m, K)]^{T}
$$

whereas $X(m, k)$ denote the symbol mapped on $k$-th subcarrier of $m$-th OFDM symbol. The symbols mapped on the $m$-th OFDM symbol $\mathbf{X}(m)=[X(1), X(2), \ldots, X(K)]^{T}$ are processed by IDFT stage to generate a time domain signal represented as

$$
\mathbf{s}=\mathbf{F}^{H} I_{N, K} \mathbf{X},
$$

here $\mathbf{F}^{H} \in \mathbb{C}^{N \times N}$ is the IDFT matrix, and $I_{N, K}$ is the resources mapper which maps the symbols to available data subcarriers. Subsequently, a CP is added to avoid ISI, then the modified signal ( $\tilde{\mathbf{s}})$ is convolved with a multipath channel and noise is added. As mentioned earlier, it is assumed that the $\mathrm{CP}$ duration is greater than the maximum excess delay of the channel, and therefore no ISI cancellation scheme is needed. The received signal $(\tilde{\mathbf{r}})$ can be written as

$$
\tilde{\mathbf{r}}=\tilde{\mathbf{s}} * \mathbf{h}+\boldsymbol{\rho}
$$

where $*$ is the linear convolution operation and $\rho$ is AWGN noise with zero mean and variance $\sigma_{\rho}^{2}$. At the receiver end, first $\mathrm{CP}$ is detached then the signal is converted to the frequency domain using the DFT stage. The symbols received at $k$-th subcarrier and $m$-th OFDM symbol can be represented as

$$
\begin{aligned}
Y(m, k)=\underbrace{H(m, k) X(m, k)}_{\text {desired signal }} \\
+\underbrace{\sum_{n=1, n \neq k}^{N} H(m, n) X(m, n)}_{\nu_{\mathrm{ICI}}(m, k)}+\underbrace{\rho(m, k)}_{\text {noise }}
\end{aligned}
$$

where $H(m, k)$ denote the channel gain at $k$-th subcarrier of $m$-th OFDM symbol and $\rho(m, k)^{2}$ is the AWGN noise with variance $\sigma_{p}$. The Doppler induced ICI observed on multipath components is represented as $\nu_{\mathrm{ICI}}(m, k)$. Finally, the symbols are equalized through an MMSE equalizer. The recovered symbol on the $k$-th subcarrier of $m$-th OFDM symbol can be represented as

$$
\begin{aligned}
\hat{X}(m, k)=W(m, k) H(m, k) X(m, k)+ \\
W(m, k)\left(\nu_{\mathrm{ICI}}(m, k)+\rho(m, k)\right)
\end{aligned}
$$

whereas $W(m, k)$ represents the MMSE equalizer matrix defined as

$$
W(m, k)=\left(\frac{\sigma_{\rho+\nu}^{2}}{\sigma_{x}^{2}}+H(m, k)^{*} H(m, k)\right)^{-1} H(m, k)^{*},
$$

whereas $\sigma_{\rho+\nu}^{2}$ is the thermal noise plus ICI variance and $\sigma_{x}^{2}$ is the transmitted symbol variance. The noise and ICI powers are assumed equally distribute on all subcarriers and subsymbols. After equalization the received symbol variance can be written as

$$
\sigma_{\hat{\mathrm{x}}}^{2}(m, k)=\left(\frac{|H(m, k)|^{2}}{|H(m, k)|^{2}+\frac{\sigma_{\rho+\nu}^{2}}{\sigma_{x}^{2}}}\right)^{2} \sigma_{x}^{2}=\alpha_{\mathrm{MMSE}}(m, k) \sigma_{x}^{2},
$$


where $\alpha_{\text {MMSE }}$ is the signal enhancement due to MMSE equalization. Similarly, noise and interference will be enhanced as

$$
\begin{aligned}
\sigma_{\rho}^{2}(m, k)=\left(\frac{|H(m, k)|}{|H(m, k)|^{2}+\frac{\sigma_{\rho+\nu}^{2}}{\sigma_{x}^{2}}}\right)^{2} \sigma_{\rho+\nu}^{2} \\
=\beta_{\mathrm{MMSE}}(m, k) \sigma_{\rho+\nu}^{2}
\end{aligned}
$$

where $\beta_{\text {MMSE }}$ is the noise and interference enhancement factor due to MMSE equalization. Now, the SINR at $k$-th subcarrier of the $m$-th OFDM symbol can be expressed as

$$
\begin{aligned}
\gamma(m, k) & =\frac{\sigma_{\hat{\mathrm{x}}}^{2}(m, k)}{\sigma_{\rho}^{2}(m, k)}=\frac{\alpha_{\mathrm{MMSE}}(m, k) \sigma_{x}^{2}}{\beta_{\mathrm{MMSE}}(m, k) \sigma_{\rho+\nu}^{2}}, \\
\gamma(m, k) & =\frac{\left(\frac{|H(m, k)|^{2}}{|H(m, k)|^{2}+\frac{\sigma_{\rho+\nu}^{2}}{\sigma_{x}^{2}}}\right)^{2} \sigma_{x}^{2}}{\left(\frac{|H(m, k)|}{|H(m, k)|^{2}+\frac{\sigma_{\rho+\nu}^{2}}{\sigma_{x}^{2}}}\right)^{2} \sigma_{\rho+\nu}^{2}}=|H(m, k)|^{2} \frac{\sigma_{x}^{2}}{\sigma_{\rho+\nu}^{2}} .
\end{aligned}
$$

The ICI power $\left(\sigma_{\nu}^{2}\right)$ at $k$-th subcarrier can be estimated using (1). It could be assumed that $\sigma_{\nu}^{2}$ is constant for all subcarriers as Doppler does not significantly vary among subcarriers in $10 \mathrm{MHz}$ bandwidth. Moreover, the signal attenuation due to resulting $\mathrm{CFO}$ can be accounted as $\mathrm{P}\left(f_{\mathrm{D}}\right)$.

\section{B. DFT-s-OFDM}

The data packet in DFT-s-OFDM can be arranged in timedomain matrix $\mathbf{x}$ with $M \times P$ dimensions, where $M$ is the size of the DFT matrix and $P$ are the number of DFT-s-OFDM symbols, given as

$$
\mathbf{x}=[\mathbf{x}(1), \mathbf{x}(2), \ldots, \mathbf{x}(P)]
$$

where

$$
\mathbf{x}(p)=[x(p, 1), x(p, 2), \ldots, x(p, M)]^{T} .
$$

The modulated symbols at each $p$-th columns are passed through DFT precoding sage leading to $\mathbf{X}=\mathbf{F}_{M} \mathbf{x}$, here $\mathbf{F}_{M} \in \mathbb{C}^{M \times M}$ denote the DFT operation. Afterward, samples are mapped to orthogonal subcarriers by means of a resource mapper $\mathbf{I}_{N, M}$ and IDFT operation $\mathbf{F}_{N}^{H}$ is performed. In this paper, $M$ equal to 16 is used such that each DFT sample is uniformly mapped to every fourth subcarrier also called interleaving. The resulting data samples can be expressed as

$$
\mathbf{s}=\mathbf{F}_{N}^{H} \mathbf{I}_{N, M} \mathbf{X} \text {. }
$$

Now, the CP is inserted at the end of each DFT-s-OFDM symbol to avoid ISI. The resulting signal ( $\tilde{\mathbf{s}})$ convolved with the channel impulse response and noise is added. The earlier made assumptions regarding $\mathrm{CP}$ duration, equalization and CFO for OFDM are also valid for the case of DFT-s-OFDM. At the receiver end, first CP is detached, then DFT operation is performed, and received samples are equalized in the frequency domain. The received samples at $p$-th DFT-s-OFDM symbol are expressed as

$$
\mathbf{Y}=\mathbf{H I}_{N, M} \mathbf{X}+\boldsymbol{\nu}_{\mathrm{ICI}}+\boldsymbol{\rho},
$$

where $\mathbf{H}=[H(1), H(2), \ldots, H(N)]$ denote the channel response at each subcarrier,

$\nu_{\mathrm{ICI}}=\left[\nu_{\mathrm{ICI}}(1), \nu_{\mathrm{ICI}}(2), \ldots, \nu_{\mathrm{ICI}}(N)\right]$ is the ICI at each subcarrier due to Doppler and $\rho$ denote the AWGN noise. The ICI variance at $k$-th subcarrier can be calculated as

$$
\nu_{\mathrm{ICI}}(k)=\sum_{n=1, n \neq k}^{N} H(n) X(n) .
$$

In the next step, samples are demapped and converted into time domain symbols using the IDFT operation. The recovered symbols at each DFT-s-OFDM symbol are represented as

$$
\hat{\mathbf{x}}=\mathbf{F}_{M}^{H} \mathbf{W H X}+\mathbf{F}_{M}^{H} \mathbf{W}\left(\boldsymbol{\nu}_{\mathrm{ICI}}+\boldsymbol{\rho}\right),
$$

whereas $\mathbf{W}$ is the MMSE equalization matrix, given as

$$
\mathbf{W}=\left(\frac{\sigma_{\rho+\nu}^{2}}{\sigma_{x}^{2}} \boldsymbol{I}_{N}+\mathbf{H}^{H} \mathbf{H}\right)^{-1} \mathbf{H}^{H}
$$

whereas $\sigma_{\rho+\nu}^{2}$ is the noise plus interference variance and $\sigma_{x}^{2}$ is the signal power. By examining (18), the received symbols at each DFT-s-OFDM symbol are $\hat{\mathbf{x}}(p)=\mathbf{F}_{M}^{H} \mathbf{W H X}$. The desired signal power of $m$-th data symbol received at $p$-th DFT-s-OFDM symbol is

$$
\begin{array}{r}
\sigma_{\hat{\mathrm{x}}}^{2}(p, m)=\left(\frac{1}{N_{m}} \sum_{k=1}^{N_{m}} \frac{|H(p, k)|^{2}}{|H(p, k)|^{2}+\frac{\sigma_{\rho+\nu}^{2}}{\sigma_{x}^{2}}}\right)^{2} \sigma_{x}^{2} \\
=\sigma_{\mathrm{MMSE}}(p, m) \sigma_{x}^{2},
\end{array}
$$

where $N_{m}$ is the data subcarriers over which $m$-th symbol is spread and $\sigma_{\text {MMSE }}$ is the desired signal amplification factor. It can be noticed that the signal power is independent of index $m$ as all data symbols spread over $N_{m}$ subcarriers will have the same amplification factor. The earlier made assumptions for OFDM regarding noise and ICI are also valid for DFT-sOFDM. As a result, the noise and ICI amplification from (18) can be obtained as

$$
\begin{array}{r}
\sigma_{\rho}^{2}(p, m)=\frac{1}{N} \sum_{k=1}^{N}\left(\frac{|H(p, k)|}{|H(p, k)|^{2}+\frac{\sigma_{\rho+\nu}^{2}}{\sigma_{x}^{2}}}\right)^{2} \sigma_{\rho+\nu}^{2} \\
=\beta_{\mathrm{MMSE}}(p, m) \sigma_{\rho+\nu}^{2}
\end{array}
$$

In addition to noise and ICI, the MMSE equalizer accumulate self-interference which can be represented as for $m$-th received symbol

$$
\begin{aligned}
& \sigma_{\mathrm{I}}^{2}(p, m)=\left(\sum_{l=1, l \neq m}^{M} \sigma_{\hat{\mathrm{x}}}^{2}(p, l)\right), \\
& \sigma_{\mathrm{I}}^{2}(p, m)=\frac{\sigma_{x}^{2}}{N_{m}} \sum_{k=1}^{N_{m}}\left(\frac{|H(p, k)|^{2}}{|H(p, k)|^{2}+\frac{\sigma_{\rho+\nu}^{2}}{\sigma_{x}^{2}}}\right)^{2} \\
& -\sigma_{\mathrm{MMSE}}(p, m) \sigma_{x}^{2} .
\end{aligned}
$$


where $M$ denotes the number of received symbols in each DFT-s-OFDM symbol. Addition and simplification of all interference terms leads to

$$
\begin{aligned}
& \sigma_{\rho}^{2}(p, m)+\sigma_{\mathrm{I}}^{2}(p, m)=\frac{\sigma_{x}^{2}}{N} \sum_{k=1}^{N} \\
& \left(\frac{|H(p, k)|^{2}\left(\frac{\sigma_{\rho+\nu}^{2}}{\sigma_{x}^{2}}+|H(p, k)|^{2}\right)}{\left(|H(p, k)|^{2}+\frac{\sigma_{\rho+\nu}^{2}}{\sigma_{x}^{2}}\right)^{2}}\right)^{2}-\sigma_{\mathrm{MMSE}}(p, m) \sigma_{x}^{2} \\
& =\sigma_{x}^{2}\left(\sqrt{\sigma_{\mathrm{MMSE}}(p, m)}-\sigma_{\mathrm{MMSE}}(p, m)\right)
\end{aligned}
$$

Finally, the SINR of $m$-th symbol received at $p$-th DFT-sOFDM symbol can be obtained as

$$
\begin{aligned}
\gamma(p, m)=\frac{\alpha_{\mathrm{MMSE}}(p, m) \sigma_{x}^{2}}{\sigma_{\rho}^{2}(p, m)+\sigma_{\mathrm{I}}^{2}(p, m)} & \\
= & \frac{1}{\left(1 / \sqrt{\alpha_{\mathrm{MMSE}}(p, m)}\right)-1}
\end{aligned}
$$

\section{GFDM}

In the case of GFDM, the data packet is arranged as a matrix $(\mathrm{X})$ of size $N \times P$, here $N=M K$, where $K$ denote the number of subcarriers, on each subcarrier $M$ symbols are mapped, and $P$ is the number of total symbols in a GFDM block, given as

$$
\mathbf{X}=[\mathbf{X}(1), \mathbf{X}(2), \ldots, \mathbf{X}(P)]
$$

where

$$
\mathbf{X}(p)=[x(p, 1), x(p, 1), \ldots, x(p, N)]^{T} .
$$

Symbols at $p$-th column are processed by a circular pulseshaping filter written as

$$
g_{k, m}[n]=g[(n-m K) \bmod N] e^{\frac{-\mathrm{j} 2 \pi \mathrm{kn}}{K}},
$$

whereas $g[n]$ denote the prototype filter, e.g., raised cosine in our case, $\bmod N$ denote the modulo $N$ operation which produces circularly shifted symbols in time and exponential part produce shifted symbols in frequency. The resulting signal is represented as

$$
\mathbf{s}=\mathbf{A} \boldsymbol{I}_{M K, M} \mathbf{X},
$$

where $I_{M K, M}$ is the resource mapper, and $\mathbf{A}=$ $\left[\boldsymbol{g}_{0,0} \cdots \boldsymbol{g}_{K-1,0}, \cdots, \boldsymbol{g}_{0, M-1} \cdots \boldsymbol{g}_{K-1, M-1}\right]$ $\in \mathbb{C}^{M K \times M K}$. After adding the CP to each GFDM block, the resulting signal ( $(\tilde{\mathbf{s}})$ passes from the multipath channel and AWGN noise is added. On the receiver, inverse processing is carried out, which includes CP removal and match filtering. The received symbols in each GFDM block can be expressed as

$$
\mathbf{Y}=\mathbf{H A} \boldsymbol{I}_{M K, M} \mathbf{X}+\boldsymbol{\nu}_{\mathrm{ICI}}+\boldsymbol{\rho},
$$

where $\mathbf{H}$ is the channel matrix with $M K \times M K$ entries, $\boldsymbol{\nu}_{\mathrm{ICI}}$ is the ICI and $\rho$ is the noise. Now, the equalized symbols can be given as

$$
\hat{\mathbf{X}}=\mathbf{W H A} \boldsymbol{I}_{M K, M} \mathbf{X}+\mathbf{A} \mathbf{W}\left(\boldsymbol{\nu}_{\mathrm{ICI}}+\boldsymbol{\rho}\right),
$$

whereas $\mathbf{W}$ is the equalization matrix of $M K \times M K$ dimensions written as

$$
\mathbf{W}=\left(\frac{\sigma_{\rho+\nu}^{2}}{\sigma_{x}^{2}} \boldsymbol{I}_{M K, M K}+(\mathbf{H A})^{H}(\mathbf{H A})\right)^{-1}(\mathbf{H A})^{H} .
$$

After following similar steps as used to drive SINR for DFTs-OFDM, the post-equalization SINR of $n$-th data symbol at $p$-th GFDM symbol is given as

$$
\gamma(p, n)=\frac{1}{\left(1 / \sqrt{\alpha_{\mathrm{MMSE}}(p, n)}\right)-1},
$$

where $\alpha_{\text {MMSE }}(p, n)$ given as

$$
\alpha_{\mathrm{MMSE}}(p, n)=\frac{1}{M N} \sum_{m=1}^{M} \sum_{n=1}^{N}\left(\frac{(\mathbf{H A})^{2}}{(\mathbf{H A})^{2}+\frac{\sigma_{\rho+\nu}^{2}}{\sigma_{x}^{2}}}\right)^{2} .
$$

\section{OTFS}

The modulated data packet $\mathbf{X}$ is arranged in the delayDoppler domain as a matrix of $N \times M$ symbols. These symbols are converted into time-frequency domain samples using ISFFT $\left(S^{H}\right)$ precoding. The ISFFT is equivalent to $2 \mathrm{D}$ DFT, one in the Doppler domain with size $M \times M$ and other in the delay domain with size $N \times N$. The resulting samples are further processed by IDFT operation $\mathbf{F}_{N}^{H}$ and the time domain signal can be expressed as

$$
\mathbf{s}=\mathbf{F}_{N}^{H} \mathbf{S}^{H} \mathbf{X}
$$

The transmitted waveform is further processed in a similar manner as mentioned earlier for other multicarrier techniques. The received symbols after performing SFFT and equalization can be written as

$$
\hat{\boldsymbol{X}}=\mathbf{S W H S}^{H} \mathbf{X}+\mathbf{S W}\left(\nu_{\mathrm{ICI}}+\boldsymbol{\rho}\right),
$$

whereas $\mathbf{W}$ represent MMSE equalization matrix of $M \times N$ dimensions written as

$$
\mathbf{W}=\left(\frac{\sigma_{\rho+\nu}^{2}}{\sigma_{x}^{2}} \boldsymbol{I}_{M, M}+\mathbf{H}^{H} \mathbf{H}\right)^{-1} \mathbf{H}^{H},
$$

where $\mathbf{H}$ is the channel response matrix of $N \times M$ dimensions. The received SINR of symbols can be obtained as

$$
\gamma=\frac{1}{\left(1 / \sqrt{\alpha_{\mathrm{MMSE}}}\right)-1}
$$

whereas $\alpha_{\text {MMSE }}$ is defined as

$$
\alpha_{\text {MMSE }}=\frac{1}{M N} \sum_{m=1}^{M} \sum_{n=1}^{N}\left(\frac{|H(m, n)|^{2}}{|H(m, n)|^{2}+\frac{\sigma_{\rho+\nu}^{2}}{\sigma_{x}^{2}}}\right)^{2} .
$$

The CP in OTFS protect symbols from both delay and Doppler impairments, if the duration in the delay domain is lager than the maximum delay spread and in Doppler domain is larger than the Doppler shift. As a result, the ICI can be easily equalized [16].

$$
P_{n} \approx \frac{1}{\sqrt{2 \pi(2 \gamma+1)}} \exp (-\gamma) .
$$


TABLE II: Optimized value of $\beta$ ( $\beta_{\text {opt }}$ ) for different multicarrier techniques

\begin{tabular}{|l|c|c|c|c|}
\hline MCS & OFDM & DFT-s-OFDM & GFDM & OTFS \\
\hline 1/2 QPSK & $-0.004 \sigma_{\bar{\gamma}}+1.86$ & $-0.016 \sigma_{\bar{\gamma}}+1.80$ & $-0.018 \sigma_{\bar{\gamma}}+1.78$ & 1.88 \\
\hline $1 / 216-\mathrm{QAM}$ & $-0.12 \sigma_{\bar{\gamma}}+8.9$ & $-0.14 \sigma_{\bar{\gamma}}+8.8$ & $-0.15 \sigma_{\bar{\gamma}}+8.6$ & 9.2 \\
\hline $3 / 4$ 16-QAM & $-0.15 \sigma_{\bar{\gamma}}+9.2$ & $-0.18 \sigma_{\bar{\gamma}}+8.9$ & $-0.19 \sigma_{\bar{\gamma}}+8.7$ & 9.4 \\
\hline 3/4 64-QAM & $-0.84 \sigma_{\bar{\gamma}}+38.4$ & $-0.92 \sigma_{\bar{\gamma}}+36.8$ & $-0.92 \sigma_{\bar{\gamma}}+36.0$ & 38.4 \\
\hline
\end{tabular}

\section{Physical Layer Abstraction for Multicarrier TECHNIQUES}

In general, PLA is used to estimate link performance as a function of the received SINR. However, the current wireless technologies use various channel coding schemes which do not have a closed-form expression for symbol error rate (SER) or PER. In addition, if the channel is time or frequency selective the received symbols inside a packet could have variable SINRs. In this case, PER depends on the average SER given as

$$
P_{\text {eff }}=\frac{1}{N} \sum_{n=1}^{N} P_{n},
$$

where $N$ denote the number of total received symbols in a packet and $P_{n}$ is the SER of $n$-th received symbol. For the case of BPSK, effective SINR ( $\left.\gamma_{\text {eff }}\right)$ from (41) can be rewritten as

$$
Q\left(\sqrt{2 \gamma_{\mathrm{eff}}}\right)=\frac{1}{N} \sum_{n=1}^{N} Q\left(\sqrt{2 \gamma_{\mathrm{n}}}\right)
$$

here $\gamma_{\mathrm{n}}$ denote the SINR of $n$-th received symbol, which could be obtained using multicarrier specific SINR expressions derived in Section IV. By observing (42), it can be said that $\gamma_{\text {eff }} \neq \frac{1}{N} \sum_{n=1}^{N} \gamma_{n}$, therefore average received SINR cannot be used to predict the average SER. Consequently, effective SINR mapping techniques are used to derive an AWGN equivalent SNR also known as effective SINR, details are provided in Appendix A. The performance of a link under fading conditions at a given effective SINR is equivalent to the performance in the AWGN channel. This equivalence allows reusing of SNR vs. PER lookup tables generated under the AWGN assumption to estimate performance under different fading conditions. According to [11], the newly proposed eEESM mapping technique outperforms EESM and RBIR and therefore will be used in this paper. It is derived by upper bounding the SER by using a tighter upper bound on error function, given by $Q(x) \approx \frac{1}{\sqrt{2 \pi\left(x^{2}+1\right)}} \exp \left(-\frac{x^{2}}{2}\right)$ [27, eq. (21)]. By using this bound, SER for BPSK [28, eq. (5.101)] can be written as

$$
P_{\text {eff }} \approx \frac{1}{\sqrt{2 \gamma_{\mathrm{eff}}+1}} \exp \left(-\gamma_{\mathrm{eff}}\right)=\frac{1}{N} \sum_{n=1}^{N} \frac{1}{\sqrt{2 \gamma_{n}+1}} \exp \left(-\gamma_{n}\right) .
$$

After solving for the effective SINR leads to the following generic expression

$$
\gamma_{\mathrm{eff}}=\frac{\beta}{2}\left\{\mathcal{W}\left[\exp (1)\left(\frac{1}{N} \sum_{n=1}^{N} \frac{1}{\sqrt{\frac{2 \gamma_{n}}{\beta}+1}} \exp \left(-\frac{\gamma_{n}}{\beta}\right)\right)^{-2}\right]-1\right\}
$$

whereas $\mathcal{W}(\cdot)$ denote the Lambert-W function given as $\mathcal{W}\left(x e^{x}\right)=x$ [29] and $\beta$ is the modulation and channel dependent parameter. The default values of $\beta$ for various modulations is as follows: 1, 2, 10, 42, and 170 for BPSK, QPSK, 16QAM, 64-QAM, and 256-QAM, respectively. These values are obtained from SER expression of these modulation schemes [11]. The optimum value of $\beta$ is obtained by using the least square fit among SER points in a fading channel such that the MSE can be minimized in reference to AWGN SER. Thus, it can be expressed as

$$
\beta_{\mathrm{opt}}=\arg \min _{\beta}\left|\gamma_{\mathrm{AWGN}}-\gamma_{\mathrm{eff}}(\beta)\right|^{2} .
$$

The traditional $\beta$ optimization depends on the MCS and channel profile. This means it needs to be tuned for each MCS against a specific channel profile and multicarrier technique. Now, if the PLA is required to be applied for a range of channel conditions with various power delay profile (PDP)s and fading distributions, either default value of $\beta$ could be used with the suboptimal performance or optimization should be carried out for all possible cases. Consequently, the optimization of $\beta$ becomes infeasible due to complexity.

To reduce optimization complexity, we model $\beta$ as a function of the received SINR variance. As variance directly depends on the channel conditions which in turn require optimization of $\beta$ to improve accuracy. Therefore, we obtain the dependency of $\beta_{\text {opt }}$ on the variance through PHY simulations and use linear regression to extend it for the general case, given as

$$
\beta=a \cdot \sigma_{\bar{\gamma}}+b
$$

where $a$ is the slope of the curve, $\sigma_{\bar{\gamma}}$ is the variance of the received SINR vector $\bar{\gamma}$ being mapped to an effective SINR and $b$ is constant. After obtaining the variables $a$ and $b$ from few channel realizations, $\beta$ can be easily generalized to other fading conditions. Moreover, this approach provides optimized $\beta$ along each SINR point of PER curve, contrary to the approach used for EESM and RBIR in literature [5], [7], where one value of $\beta$ is used to fit all SINR points along the PER curve. In Table II optimized value of $\beta$ are provided for different MCSs and multicarrier techniques. The reason behind $a=0$ in the case of OTFS is due to time and frequency domain spreading of symbols which results in equal SINR inside an OTFS block.

The concept of modeling end-to-end PHY performance of considered multicarrier techniques using PLA is illustrated in Fig. 4. The symbol SINR expressions derived in the previous section for different multicarrier techniques are used here. Based on the channel selectivity symbol SINRs are estimated in a packet. If variable SINRs are expected, depending on the 


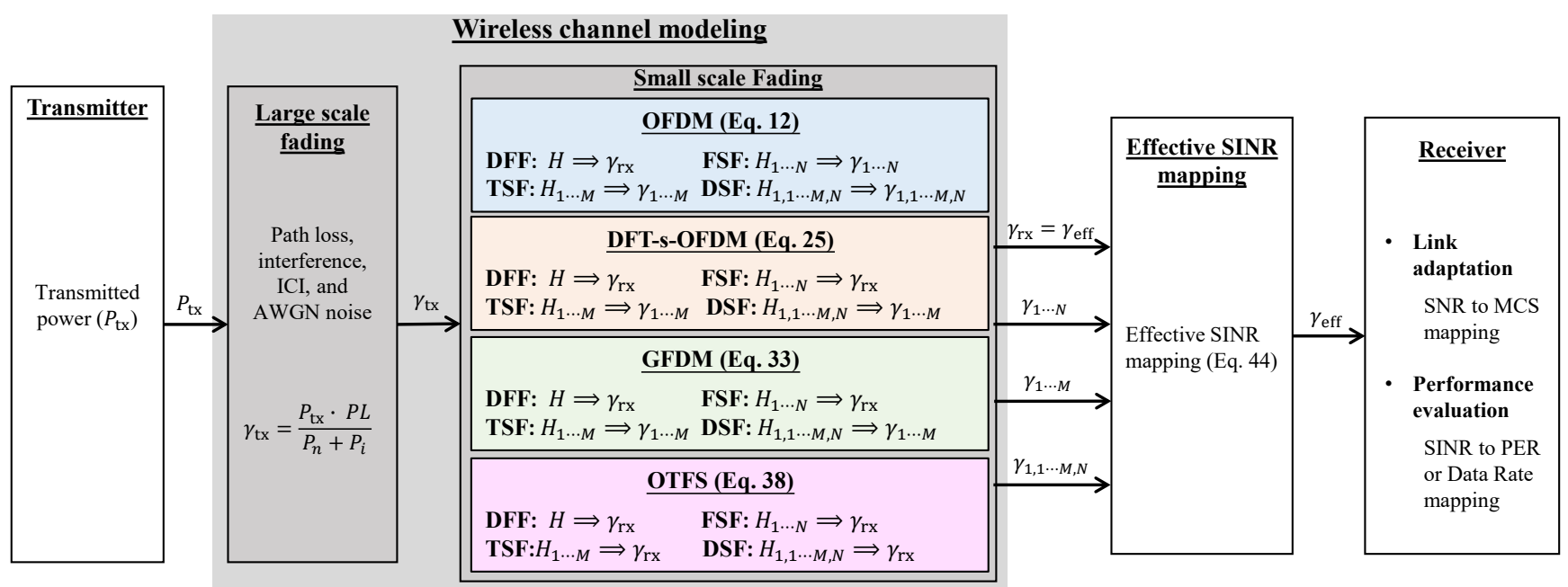

Fig. 4: PLA modeling for multicarrier modulation techniques

multicarrier technique and fading conditions, effective SINR is calculated using (44). The effective SINR is then assumed as AWGN equivalent SNR and pre-computed lookup tables are used to estimate link performance.

In the case of OFDM, each data symbol is mapped to a unique subcarrier for the duration of $T_{s}$. Therefore, under frequency or time selective fading symbols could experience variable channel gain. If the channel is selective, its gain could be either represented with a vector, as in the case of FSF or time selective fading (TSF), or with a matrix, as in the case of DSF. Consequently, the received packet has multiple SINRs, which should be mapped to an effective SINR using (44). On the other hand, if the channel is doubly flat fading (DFF), effective SINR mapping is not needed. Since all symbols inside a packet will have equal SINR. As a result, the channel is represented with a single gain, and the received SINR obtained using (12) will serve as an effective SINR. Contrary to OFDM, data symbols in DFT-s-OFDM are spread over the frequency domain. If received symbols inside a packet are mapped at the same subcarriers indexes then effective SINR computation is not needed for FSF channel, as all received symbols will have equal SINR. However, if data symbols are transmitted over different groups of subcarriers while mapping $M$ DFT samples to $N$ available subcarriers ( $N / M$ groups can be made), effective SINR will be calculated among these groups. Nevertheless, for the case of DSF channel SINR mapping is also required among DFT-s-OFDM symbol blocks for which channel is not considerably flat. For the other two cases, DFF and TSF, an effective SINR mapping is similar to OFDM. In GFDM, the $N$ data symbols are spread over $k$ subcarrier and $M$ subsymbols. Therefore, under FSF or DFF channel SINR mapping is not required, similar to DFT-sOFDM. Contrarily, under DSF or TSF fading SINR mapping could be required among GFDM blocks, depending on the relation between coherence time and packet duration. The data packet in OTFS is commonly transmitted in one transversal block, which means data symbols are equally spread over time and frequency. In this case, no SINR mapping is required and the received SINR is used for performance evaluation.
However, if data is transmitted over multiple transversal blocks then effective SINR mapping would be required among these blocks. After having the effective SINR, the performance of any multicarrier technique could be estimated by utilizing AWGN lookup tables.

\section{EVAluation OF MULTICARRIER PLA}

In this section, multicarrier modulation-specific PHY simulators are used to validate the accuracy of developed PLA. On one hand, PER is calculated using a complete PHY chain, on the other hand, performance is predicted using the multicarrier specific PLA (as explained in the previous section). The fading channel between users is modeled either as FSF or DSF using the PDP given in Table III. The ideal channel estimation and CFO correction are assumed. Other relevant parameters for simulations are available in Table III. The PLA validation is carried out here in two steps: accuracy of effective SINR mapping in reference to performance in AWGN channel, and PLA prediction accuracy compared to PHY simulations in a fading channel.

\section{A. Accuracy of Effective SINR Mapping}

The purpose of this investigation is to validate the accuracy of PLA against AWGN curves. For this purpose, the effective SINR vs. PER performance in fading channel is obtained through simulations and compared with AWGN reference curves and error is calculated. In the case of a significant mapping error, optimization of $\beta$ should be carried out for each MCS, such that the error between both SINRs (i.e., AWGN and effective SINR for the same value of PER) could be minimized. In Fig. 5, the PER of different MCSs is plotted in AWGN channel, represented with solid lines. Moreover, the effective SINR vs. PER performance in FSF channel is also plotted for multicarrier modulations, represented by markers. It is important to mention that these results are obtained with optimized values of $\beta$ listed in Table II. The results show perfect overlapping between PER of different multicarrier techniques obtained in a fading channel and PER 
TABLE III: Simulation Parameters

\begin{tabular}{|c|c|}
\hline Parameter & Value \\
\hline \multicolumn{2}{|c|}{ PHY settings } \\
\hline Channel bandwidth & $10 \mathrm{MHz}$ \\
\hline FFT length & 64 (others), 16 for GFDM $(\mathrm{K}=16, \mathrm{M}=4)$ \\
\hline Carrier Spacing & $156.25 \mathrm{KHz}$ (others), $625 \mathrm{KHz}$ (GFDM) \\
\hline Symbol duration & $6.4 \mu \mathrm{s}$ \\
\hline Cyclic prefix & $1.6 \mu \mathrm{s}$ \\
\hline Payload size $\left(P_{b}\right)$ & 300 bytes \\
\hline Modulation and coding schemes (MCS) & given in Table II \\
\hline $\begin{array}{l}\text { Channel coding } \\
\text { Packet duration }\left(T_{p}\right) \text { for considered MCSs }\end{array}$ & $\begin{array}{c}\text { LDPC (fifth generation }(5 \mathrm{G}) \text { new radio }(\mathrm{NR}) \text { ) } \\
{[304,152,104,72] \mu \mathrm{s}}\end{array}$ \\
\hline Channel equalization method & MMSE \\
\hline \multicolumn{2}{|c|}{ Small scale fading } \\
\hline Maximum doppler shift $\left(f_{\mathrm{m}}\right)$ & $100 \mathrm{~Hz}(\mathrm{FSF}) / 2000 \mathrm{~Hz}$ (DSF) \\
\hline Relative power of each tap & {$\left[\begin{array}{llll}0 & -10 & -14 & -18\end{array}\right] \mathrm{dB}$} \\
\hline Excess delay of each tap & {$\left[\begin{array}{llll}0 & 0.4 & 0.7 & 1\end{array}\right] \mu \mathrm{s}$} \\
\hline Coherence time $(50 \%)$ & $4.23 \mathrm{~ms}$ (FSF), $0.21 \mathrm{~ms}$ (DSF) \\
\hline Coherence bandwidth (50\%) & $\approx 1 \mathrm{MHz}$ \\
\hline Fading distribution & Rayleigh \\
\hline
\end{tabular}

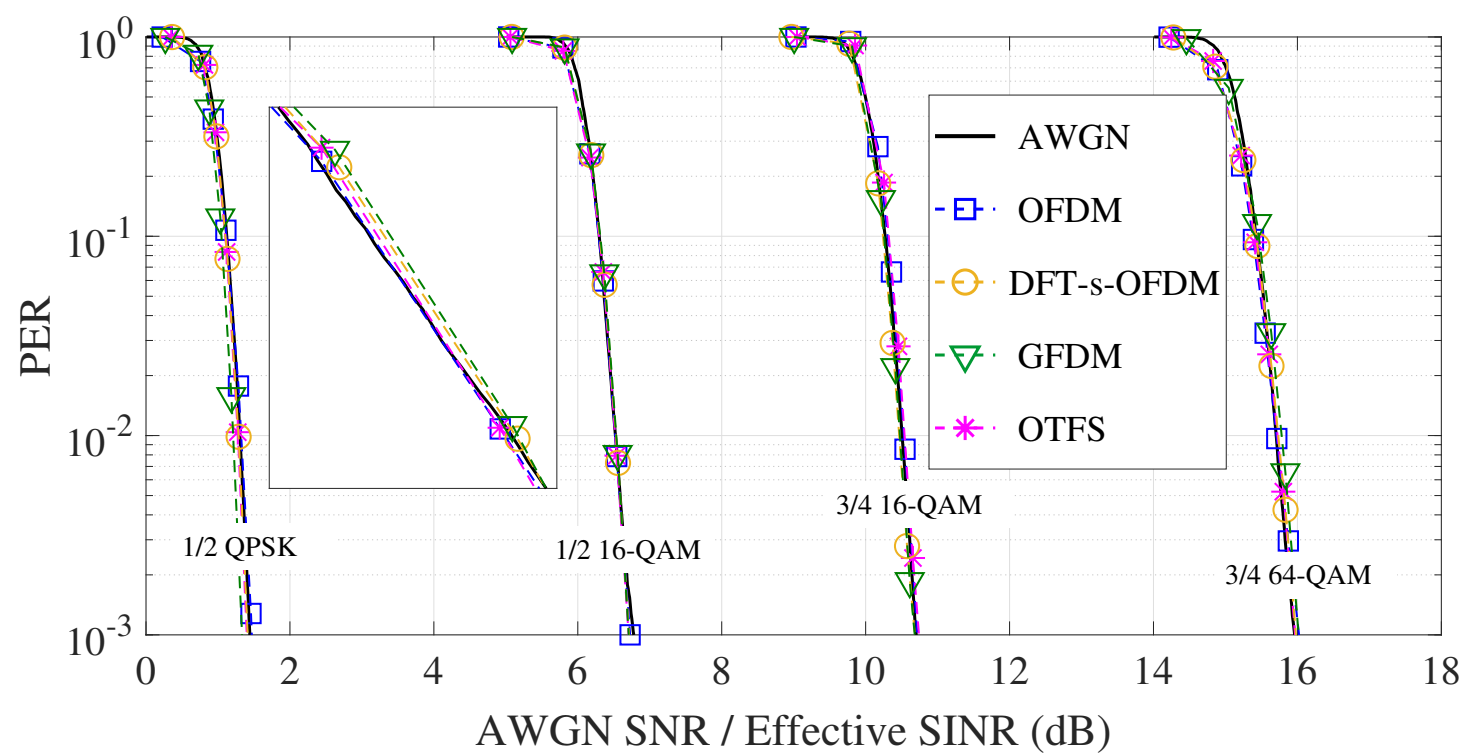

Fig. 5: The PER performance in terms of effective SINR

in the AWGN channel. As explained in the previous section that ideally, the performance of a fading channel as a function of effective SINR should have a similar performance as in the AWGN channel. If this holds, a single lookup table per MCS could be used to predict the performance of various multicarrier techniques in a range of fading scenarios. This simplifies system-level simulation as well as link adaptation. From the results, it could be concluded that the performance of all multicarrier modulations as a function of the effective SINR is almost equivalent.

The overlapping PER curves of multicarrier modulations in Fig. 5 does not reflect that all modulation techniques have similar performance in terms of transmitted SINR. The transmitted SINR here $\left(\gamma_{\mathrm{tx}}\right.$ in Fig. 4) means that signal is transmitted with defined transmit power and interference-plusnoise ratio. In practice various techniques may have different effective SINR, even though, the transmitted SINR is equal. This is illustrated in Fig. 6, where average effective SINR vs. transmitted SINR of different multicarrier techniques is plotted for FSF and DSF channels with 1/2 16-QAM. Results show that the OTFS outperforms other techniques in terms of effective SINR due to the spreading of signals in both time and frequency domains. Moreover, its effective SINR is least dependent on the channel response. In the case of FSF channel, DFT-s-OFDM and GFDM have comparable performance to OTFS due to similar diversity gain. By contrast, effective SINR difference increases in DSF channel, because OTFS has additional diversity gain due to time selectivity. Comparing DFT-s-OFDM and GFDM, it can be noticed that effective SINR of DFT-s-OFDM is slightly higher for the case of FSF channel and marginally lower for the case of DSF channel (i.e., when transmitting SINR $>20 \mathrm{~dB}$ ). The better performance of 


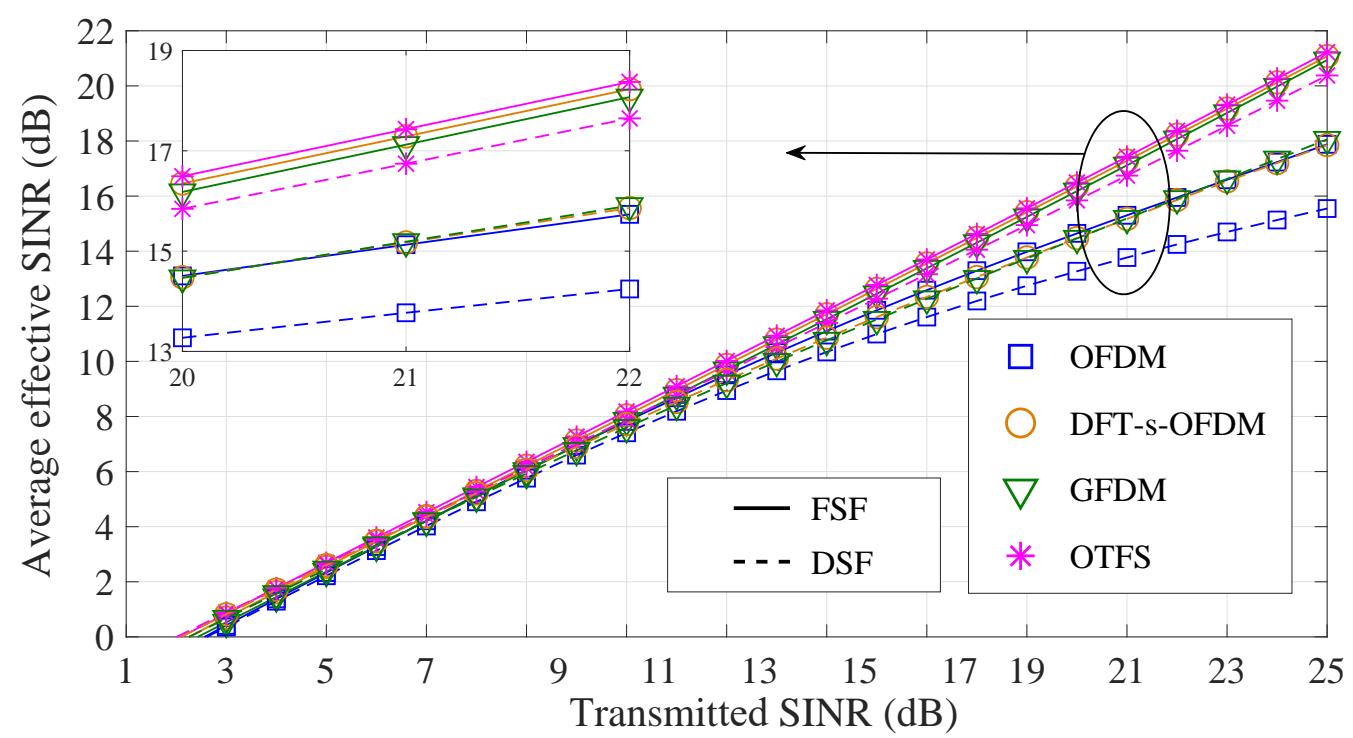

Fig. 6: Effective SINR of various multicarrier modulations under fading conditions (1/2 16QAM)

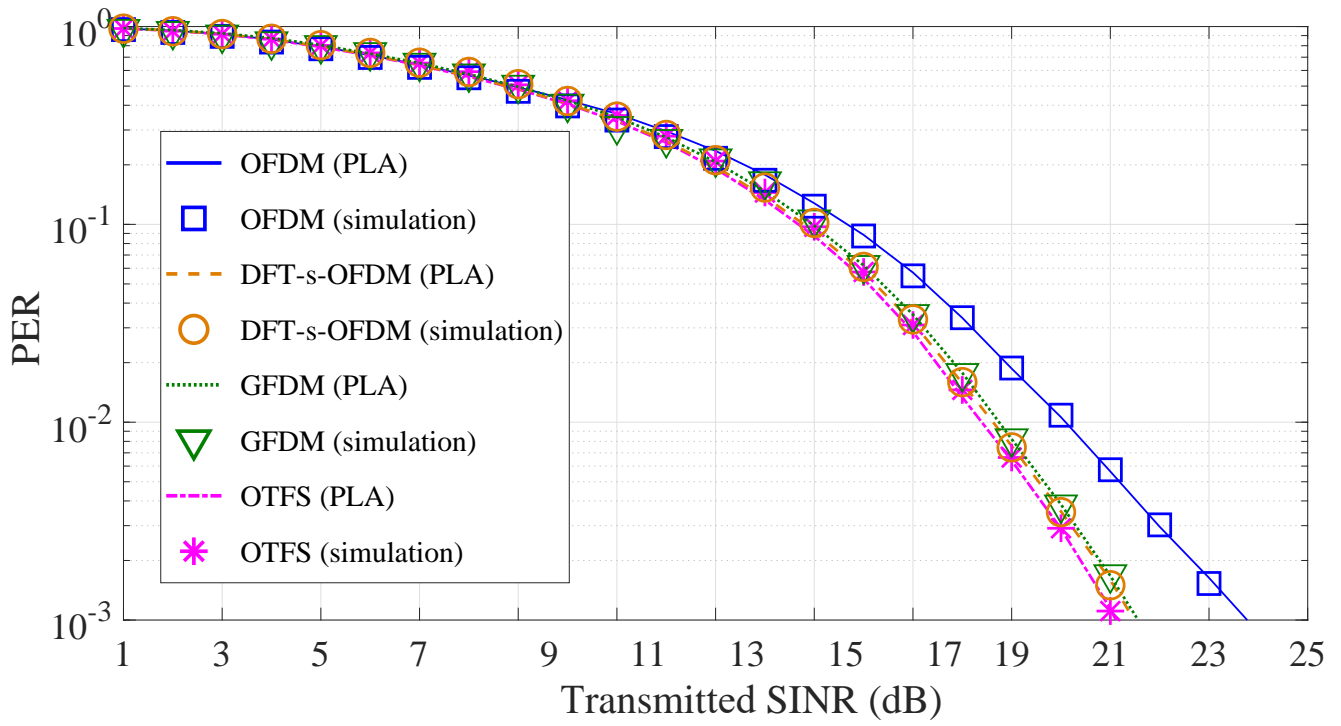

Fig. 7: Estimated and simulated PER of multicarrier modulation techniques under DSF channel

DFT-s-OFDM is due to higher diversity gain from interleaved subcarriers with 4 times lower subcarrier spacing compared to GFDM. In the presence of Doppler lower subscriber spacing result in higher ICI which degrade its performance, as in the case of DSF channel. Nevertheless, OFDM gives the least value of effective SINR compared to other techniques, and the difference increases with channel selectivity. The reason for this is the independent fading experienced by received data symbols in OFDM, where PER is dominated by deep fades and results in lower values of effective SINR. Conversely, in the case of other modulations, deep nulls are averaged out due to the spreading of data in either frequency domain (such as DFT-s-OFDM and GFDM) or in both time and frequency domain (such as OTFS).

\section{B. Prediction Accuracy of PLA}

The previous subsection shows that the performance of all multicarrier techniques at a certain effective SINR is equiv- alent to AWGN SNR. Consequently, lookup tables generated under the AWGN assumption can be used for performance estimation in fading channels. To demonstrate this, PER performance of multicarrier modulations is estimated in a DSF channel. The estimated and simulated PER values are plotted in Fig. 7 for comparison. Once again results show a good match between predicted and simulated performance for all considered multicarrier techniques. By comparing the performance among multicarrier modulations, a similar trend to Fig. 6 can be observed. An important point to be noted is that Fig. 6 and Fig. 7 show different comparison. The Fig. 6 depict gain in terms of average effective SINR, whereas Fig.7 compare average PER, estimated based on the instantaneous effective SINR. Due to the fact that both gains are not interchangeable, the difference in terms of transmitted SINR is not equal. Furthermore, it is also worth mentioning that the real performance gain of OTFS and DFTs-OFDM can only be achieved through iterative receivers, which cancel out the ISI 


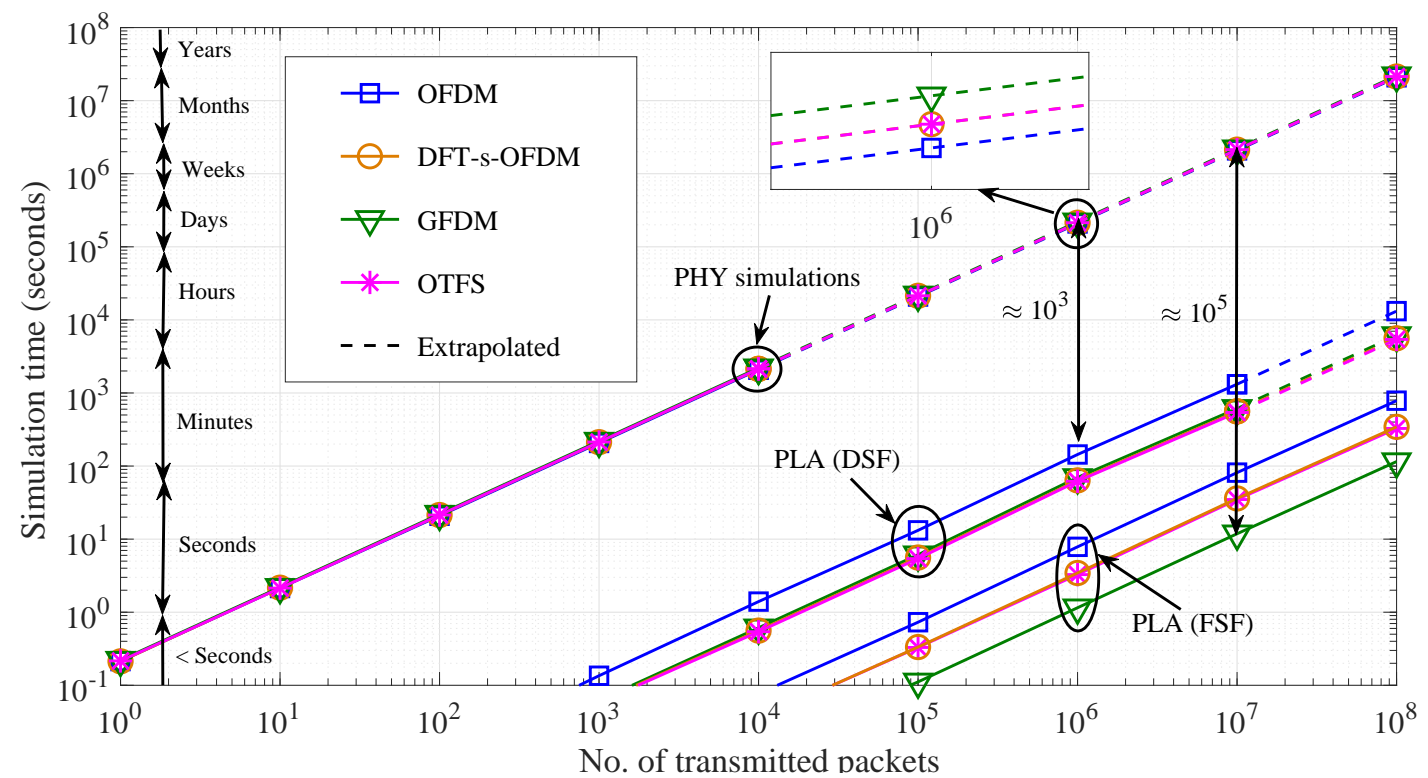

Fig. 8: Comparison between PHY simulation and PLA in terms of simulation time (1/2 16-QAM)

among data symbols introduced by channel excess delay.

\section{Simulation Complexity Analysis}

In order to give an insight about the reduction in computational complexity by using PLA instead of PHY simulations, a comparison is provided in Fig 8. The computation time of PHY simulations is almost the same for different multicarrier techniques because they go through similar processing except additional precoding required in the case of DFT-s-OFDM, GFDM, and OTFS. Therefore, OFDM achieves the lowest simulation time followed by OTFS and DFT-s-OFDM due to DFT and SSFT precoding, respectively. The GFDM requires the highest simulation time due to DFT processing with circular pulse shaping. However, the simulation time of OTFS, GFDM, and DFT-s-OFDM will further increase if the iterative receiver will be used. Now comparing the computation time of multicarrier techniques when PLA is used, the opposite trend is observed compare to PHY simulation. First considering the DSF channel, PLA of OFDM requires mapping in both time and frequency domain and therefore requires more computation time, whereas, DFT-s-OFDM and GFDM require mapping in the time domain and OTFS does not require mapping at all. Even though effective SINR computation is not needed in the case of OTFS, it still needs to calculate receive SINR from $M \times N$ channel matrix. Further, looking at the FSF channel, only OFDM requires effective SINR mapping, whereas other techniques only require to compute receive SINR from the frequency domain matrix, which is $1 \times N$ for the case of OTFS and DFT-s-OFDM and $1 \times K$ for GFDM. Finally, comparing the PHY simulation with PLA, simulation time can be reduced by a factor of $10^{3}$ in the worst-case scenario, which is OFDM in the DSF channel, and $10^{5}$ in the best-case scenario, which is GFDM in the FSF channel. Hence, it can be concluded that PLA is an effective method to speed up system-level simulations and to evaluate higher-order probabilities within an affordable time.

\section{COMPARISON OF Multicarrier Modulation TECHNIQUES}

In this section, a comparison of modern multicarrier modulation techniques is provided under various fading conditions. Such comparison is generally required to evaluate the pros and cons of a newly proposed technique in reference to the existing ones. In order to compare the performance of multicarrier techniques, a simple system-level simulator is considered with one transmitter and receiver. The concept of link-level to the system-level interface is illustrated in Fig. 4. At the system level, the arbitrary frequency response of the channel is generated depending on the PDP or fading conditions. The PDP and Doppler shift values for the considered fading conditions (i.e., DFF, TSF, FSF, and DSF) are provided in Table III. The carrier spacing for all multicarrier techniques is assumed the same except GFDM ( $M$ times higher, where $M$ is the number of subsymbols in GFDM block). For comparison, one lower-order MCS, i.e., 1/2 QPSK, and one higher-order MCS, i.e., 3/4 64 QAM, are selected. In the case of time selective channels, performance is evaluated with and without ICI (assuming an ideal ICI cancellation scheme).

Doubly Flat Fading (DFF): In the case of DFF, the channel is assumed flat in both time and frequency. This type of fading is commonly experienced in indoor environments, having strong LOS components, few week multipath components, smaller excess delay, and relatively static or slower user speed. Consequently, the choice of carrier spacing and ICI is not relevant $\left(\tau_{s} \approx 0\right)$. The Channel gain, in this case, could be represented by $\mathbf{H}$ with a single entry. The PER of various multicarrier techniques is shown in Fig. 9(a) for the DFF channel. As all symbols encounter same fading conditions irrespective of the used multicarrier technique hence all multicarrier technique have similar performance. Therefore, the choice of a multicarrier technique does not affect performance.

Frequency Selective Fading (FSF): For the case of FSF, the channel is assumed flat in time but selective in frequency. 


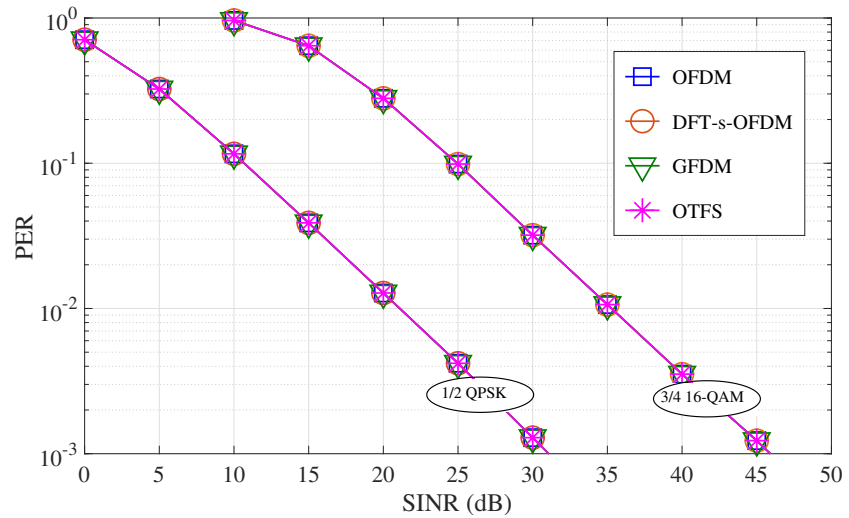

(a) DFF

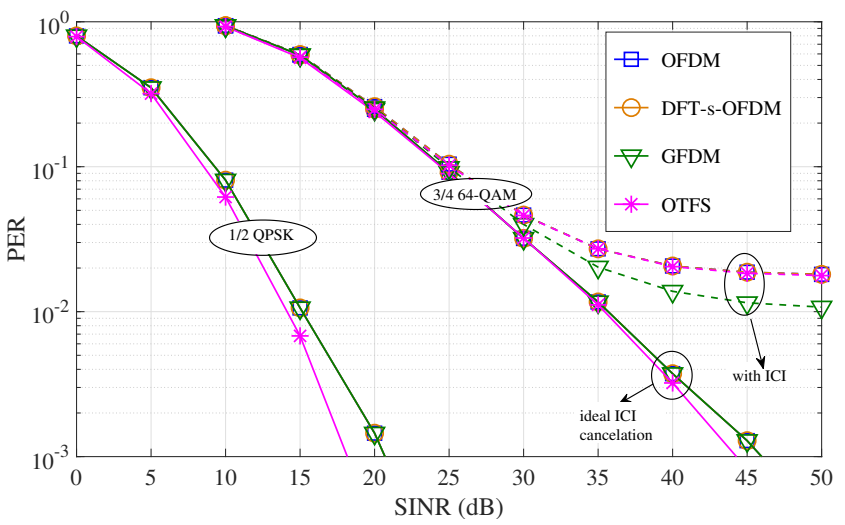

(c) TSF

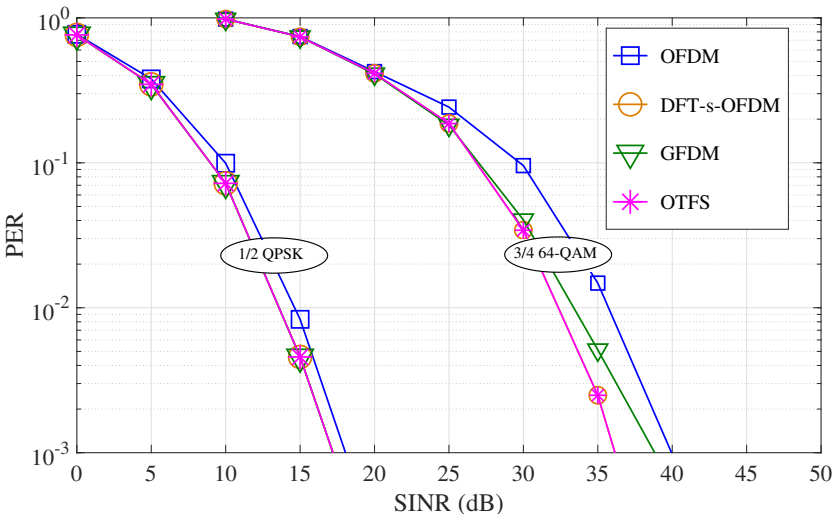

(b) FSF

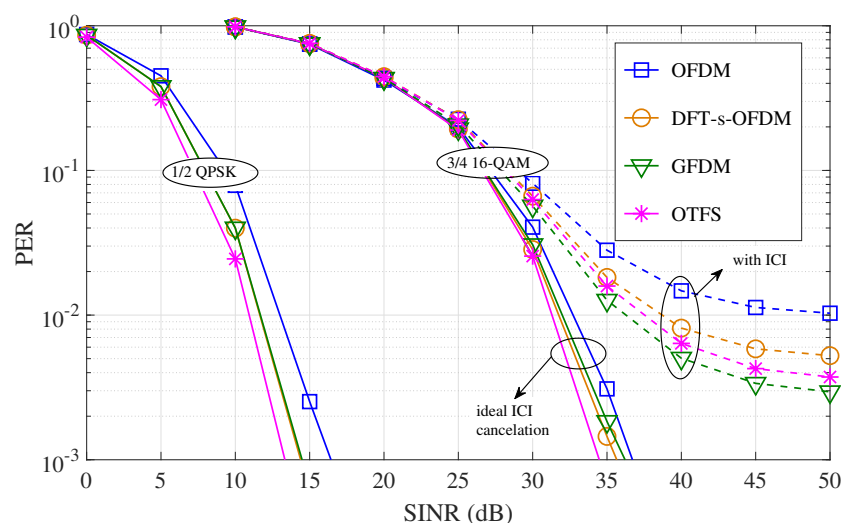

(d) DSF

Fig. 9: Performance comparison of multicarrier modulation techniques

The FSF is encountered in large indoor halls or in outdoor environments, having strong multipath components, large excess delay, and relatively static or slower user speed. Similar to DFF, ICI is not relevant $\left(\tau_{s} \approx 0\right)$. The channel gain for this case is represented by $\mathbf{H}$ having $1 \times N$ entries, where $N$ is the number of subcarriers. The received SINR per block could be obtained using the SINR expressions followed by effective SINR mapping. The PER of considered multicarrier schemes is shown in Fig. 9(b) for the FSF channel. Both, OTFS and DFTs-OFDM deliver almost similar PER performance because they spread symbols in the frequency domain and therefore achieve higher energy per bit compared to OFDM. The GFDM also spread data symbols in the frequency domain, however, with four times less number of subcarriers. Furthermore, the difference in performance among multicarrier techniques is higher for the case of 3/4 64-QAM compared to $1 / 2$ QPSK. This is due to the fact that with the increase in modulation order more bits are transmitted in one OFDM symbol. Therefore, more number of bits experience frequency diversity. Furthermore, the performance of all multicarrier techniques is relatively better compared to the DFF channel due to the frequency diversity.

Time Selective Fading (TSF): If the channel response is flat in frequency but selective in time, it is known as TSF channel. In Section III, time selectivity is defined by $\tau_{s}$, its value close to 1 denote highly selective channel and its value close to 0 represent flat channel. Alternatively, a ratio between coherence time and packet duration could also be used. The TSF channel is usually experienced in outdoor environments, having LOS communication, fewer week multipath components, smaller excess delays, and users moving at high speed. For the used carrier spacing (i.e., $156.25 \mathrm{kHz}$ ), the ICI power is only dominated for SINR higher than $30 \mathrm{~dB}$ (Table I, Fig. 2). Therefore, comparison with and without ICI cancellation is only shown for 3/4 64-QAM, as it has a negligible effect on the 1/2 QPSK performance. The TSF channel could be abstracted with $\mathbf{H}$ having $1 \times P$ entries, where $P$ are the total number of blocks in time such that in each block channel could be assumed flat. The received SINR per block could be obtained using the SINR expressions followed by effective SINR mapping. Results for this case are plotted in Fig. 9(c). For ideal ICI cancellation case, it could be observed that except OTFS all other techniques have almost similar performance. This is due to the spread of symbol energy over the packet duration in the case of OTFS, which results in higher receive energy per symbol in TSF. Comparing the gain of OTFS between MCSs, $1 / 2$ QPSK has a slightly higher gain compared to 3/4 64-QAM due to the longer packet duration. In the presence of ICI, OTFS performs marginally better compared to OFDM and DFT-sOFDM, as performance is now ICI dominated. Interestingly, due to higher carrier spacing in GFDM, it outperforms all other multicarrier techniques as it results in lower ICI. Similar to the case of FSF, all multicarrier techniques perform considerably better as compared to the DFF channel. This gain is due to time diversity given that ICI could be ideally equalized. It must be noted that for practical systems, channel estimation error 
increases with the decrease in coherence time, which results in performance degradation instead of diversity gain. As ideal channel estimation is assumed here, hence these effects could not be observed from results.

Doubly Selective Fading (DSF): In DSF, the channel changes rapidly in both time and frequency. Such fading conditions are observed in outdoor environments, having strong multipath components, large multipath excess delays, and users moving at high speed. The eV2X communication scenarios fall generally in this category, such as Urban Crossing NLOS, Highway LOS, and Highway NLOS [18]. Similar to TSF, ICI impacts link performance and needs to be considered. To abstract the DSF channel, $\mathbf{H}$ with $P \times N$ entries is required. The received SINR of each subcarrier in a block could be obtained using the SINR expressions followed by two-fold effective SINR mapping (one in frequency and the other in time domain). The PER performance of multicarrier techniques is depicted in Fig. 9(d). For the case of ideal ICI cancellation, OTFS has superior performance compared to other multicarrier techniques. This is due to the spread of symbols in both the time and frequency domain which results in higher diversity gain compared to other techniques. Similar to TSF, OTFS gain is slightly higher for $1 / 2$ QPSK compared to 3/4 64-QAM due to longer packet duration. The performance of DFT-s-OFDM is slightly lower than OTFS as it only spread symbols in the frequency domain. The GFDM has a slightly lower gain compared to DFT-s-OFDM due to 4 times less number of subcarriers. The worst performing multicarrier technique is OFDM due to variable SINR per each data symbol. In the presence of ICI, OTFS outperforms OFDM and DFT-s-OFDM due to inherent time and frequency diversity, although GFDM has slightly better performance due to higher carrier spacing. Overall, multicarrier techniques have better performance compared to other fading scenarios if ICI could be equalized. This is due to the higher time and frequency diversity experienced by symbols.

Concluding Remarks: The presented comparison can be summarized as:

- In the DFF channel, no gain over a single carrier could be achieved by using any multicarrier technique due to flat fading.

- Generally, the PER of all multicarrier techniques decreases with the increase in either time or frequency selectivity, given that, the ideal channel estimation, ICI and ISI cancellation is possible.

- Overall OTFS performs better compared to other multicarrier techniques in all evaluated scenarios, although the gain is higher in the DSF channel.

- In eV2X communication scenarios performance is mainly ICI limited. Consequently, multicarrier technique with easy ICI cancellation scheme suits best, as claimed for OTFS [2].

- Comparing both complexity and performance following is true: OTFS is the best suited for time-varying channels (such as DSF and TSF), DFT-s-OFDM for the FSF channel, and GFDM for ICI dominated scenarios.

\section{CONCLUSIONS}

In this paper, the modern multicarrier modulation schemes are considered for future wireless communications systems. The received SINR of these multicarrier techniques is derived for the frequency domain MMSE equalization and fading specific PLA is proposed. The accuracy of the proposed PLA is validated through full PHY simulations. Results demonstrate that PLA can precisely estimate PHY performance under various fading conditions. The performance evaluation in terms of effective SINR shows that OTFS deliver higher effective SINR compared to other techniques. The reason for the better performance of OTFS is the spread of symbol energy over time and frequency, which results in higher effective SINR due to higher diversity. Comparison in terms of simulation time shows that PLA for all multicarrier techniques provides a significant gain in terms of simulation time compared to PHY simulations. Multicarrier techniques which spread data symbols in time/frequency have lower simulation time compared to OFDM, which is because fewer SINRs are left to calculate effective SINR. Overall, a gain of $\approx 10^{3}$ could be achieved for DSF channel and a gain of $10^{5}-10^{6}$ for FSF channel.

The performance comparison among multicarrier techniques shows that in the DFF channel the choice of multicarrier modulation does not affect PER performance. This is due to the fact that all symbols experience similar fading irrespective of the selected technique. Overall OTFS outperforms all other multicarrier techniques in the DSF channel and has comparable performance to DFT-s-OFDM in the FSF channel. Considering complexity and performance jointly, OTFS is the best-suited multicarrier modulation for time varying channels (such as DSF and TSF), DFT-s-OFDM and GFDM for the FSF channel, and OFDM for DFF channel.

\section{APPENDIX A}

\section{EFFECTIVE SINR MAPPING TECHNIQUES}

\section{A. Exponential Effective SINR Mapping (EESM)}

The EESM expression is obtained by upper bounding the symbol error probability with Chernoff bound [30]. The generic effective SINR $\left(\gamma_{\mathrm{eff}}\right)$ mapping for different modulation schemes can be obtained as [5]

$$
\gamma_{\mathrm{eff}}=-\beta \ln \left(\frac{1}{N} \sum_{n=1}^{N} \exp \left(-\frac{\gamma_{n}}{\beta}\right)\right) .
$$

The default values of $\beta$ are the same as in the case of eEESM and can be optimized using (45).

\section{B. Received Bit Information Rate (RBIR)}

The effective SINR in case of RBIR is obtained by first calculating the mutual information per received symbol and then mapping back the mean mutual information to SINR [6]. The effective SINR in the case of RBIR can be calculated as

$$
\gamma_{\text {eff }}=\beta \Phi^{-1}\left\{\frac{1}{N} \sum_{n=1}^{N} \Phi\left(\frac{\gamma_{n}}{\beta}\right)\right\},
$$


where $\Phi$ denote the mean mutual information of the received symbols, given as

$$
\begin{aligned}
\Phi(\gamma, M)=\log _{2}(M) & -\frac{1}{M} \sum_{m=1}^{M} E_{U}\left\{\operatorname { l o g } _ { 2 } \left(\sum _ { k = 1 } ^ { M } \operatorname { e x p } \left(|U|^{2}\right.\right.\right. \\
& \left.\left.\left.-\left|\sqrt{\gamma}\left(s_{k}-s_{m}\right)+U\right|^{2}\right)\right)\right\},
\end{aligned}
$$

whereas $U$ is a random Gaussian variable with zero mean and unit variance, $E_{U}$ represent the expectation of $U, s_{k}$ denote the $k$-th and $s_{m}$ denote the $m$-th constellation points, and $M$ represent the total number of bits carried by the used modulation scheme. Similar to EESM, $\beta$ is the optimization parameter and its default value for all modulation schemes is equal to 1 , whereas optimized values can be obtained using (45).

\section{Enhanced EESM (eEESM)}

As mentioned earlier, the eEESM expression is derived by using another tighter bound on SER [11]. The missing derivation steps from Section $\mathrm{V}$ are given here.

After applying log and some mathematical manipulations of (43) leads to

$$
\log \left(2 \gamma_{\mathrm{eff}}+1\right)+2 \gamma_{\mathrm{eff}}=-2 \log \left(\frac{1}{N} \sum_{n=1}^{N} \frac{1}{\sqrt{2 \gamma_{n}+1}} \exp \left(-\gamma_{n}\right)\right) .
$$

After adding 1 and applying exponent on both side, we get

$$
\left(2 \gamma_{\text {eff }}+1\right) \exp \left(2 \gamma_{\text {eff }}+1\right)=\left(\frac{1}{N} \sum_{n=1}^{N} \frac{1}{\sqrt{2 \gamma_{n}+1}} \exp \left(-\gamma_{n}\right)\right)^{-2} \exp (1) \text {. }
$$

Now, solving for $\gamma_{\mathrm{eff}}$, we get

$$
\gamma_{\text {eff }}=\frac{1}{2}\left\{\mathcal{W}\left[\exp (1)\left(\frac{1}{N} \sum_{n=1}^{N} \frac{1}{\sqrt{2 \gamma_{n}+1}} \exp \left(-\gamma_{n}\right)\right)^{-2}\right]-1\right\} \text {. }
$$

A generic expression for eEESM is given in (44).

\section{REFERENCES}

[1] W. Anwar, A. Kumar, N. Franchi, and G. Fettweis, "Performance analysis using physical layer abstraction modeling for $5 \mathrm{G}$ and beyond waveforms," in IEEE Global Communications Conference (IEEE Globecom), Waikoloa, Hawaii, USA, Dec. 2019.

[2] T. Zemen, M. Hofer, and D. Loeschenbrand, "Low-complexity equalization for orthogonal time and frequency signaling (OTFS)," CoRR, vol. abs/1710.09916, 2017.

[3] Hadani et al., "Orthogonal time frequency space modulation," in IEEE Wireless Communications and Networking Conference (WCNC), Mar 2017.

[4] Mesa et al., "Link abstraction models for multicarrier systems: A logistic regression approach," International Journal of Communication Systems, vol. 31, Sep. 2017.

[5] J. Olmos, A. Serra, S. Ruiz, M. García-Lozano, and D. Gonzalez, "Exponential effective SIR metric for LTE downlink," in IEEE 20th International Symposium on Personal, Indoor and Mobile Radio Communications, Sep. 2009.

[6] R. Hoefel and O. Bejarano, "On application of PHY layer abstraction techniques for system level simulation and adaptive modulation in IEEE 802.11ac/ax systems," Journal of Communication and Information Systems, vol. 31, pp. 198-210, Jan. 2016.
[7] J. Wu, Z. Yin, J. Zhang, and W. Heng, "Physical layer abstraction algorithms research for 802.11n and LTE downlink," in International Symposium on Signals, Systems and Electronics, Sep. 2010.

[8] J. Francis and N. B. Mehta, "EESM-based link adaptation in pointto-point and multi-cell OFDM systems: Modeling and analysis," IEEE Transactions on Wireless Communications, vol. 13, no. 1, pp. 407-417, Jan. 2014.

[9] W. Anwar, K. Kulkarni, N. Franchi, and G. Fettweis, "Physical layer abstraction for ultra-reliable communications in $5 \mathrm{G}$ multi-connectivity networks," in IEEE 29th Annual International Symposium on Personal, Indoor and Mobile Radio Communications (PIMRC), Sep. 2018.

[10] W. Anwar, S. Dev, K. Kulkarni, N. Franchi, and G. Fettweis, "On PHY abstraction modeling for IEEE 802.11ax based multi-connectivity networks," in IEEE Wireless Communications and Networking Conference $(W C N C)$, Apr. 2019.

[11] W. Anwar, S. Dev, A. Kumar, N. Franchi, and G. Fettweis, "PHY abstraction techniques for V2X enabling technologies: Modeling and analysis," IEEE Transactions on Vehicular Technology, Feb 2020.

[12] W. Anwar, A. Kumar, N. Franchi, and G. Fettweis, "Physical layer abstraction for multi-connectivity communications: Modeling and analysis," IEEE Transactions on Wireless Communications, Aug. 2021.

[13] W. Anwar, A. Krause, A. Kumar, N. Franchi, and G. Fettweis, "Performance analysis of various waveforms and coding schemes in V2X communication scenarios," in IEEE Wireless Communications and Networking Conference (WCNC), Seoul, Korea, Apr. 2020.

[14] Gerzaguet et al., "The 5G candidate waveform race: a comparison of complexity and performance," EURASIP Journal on Wireless Communications and Networking, vol. 1, p. 13, Jan 2017.

[15] Matthé et al., "Generalized frequency division multiplexing: A flexible multi-carrier waveform for 5G," $5 G$ Mobile Communications, pp. 223 259, Oct. 2017.

[16] R. Hadani and A. Monk, "OTFS: A new generation of modulation addressing the challenges of 5G," CoRR, vol. abs/1802.02623, 2018.

[17] W. Anwar, N. Franchi, and G. Fettweis, "Physical layer evaluation of V2X communications technologies: 5G NR-V2X, LTE-V2X, IEEE 802.11bd, and IEEE 802.11p," in IEEE Vehicular Technology Conference (VTC Fall), Honolulu, Hawaii, USA, Sep 2019.

[18] M. Kahn, "V2V radio channel models," IEEE 802.11-14/0259r0, Feb. 2014.

[19] Peng Tan and N. C. Beaulieu, "Reduced ICI in OFDM systems using the "better than" raised-cosine pulse," IEEE Communications Letters, vol. 8, no. 3, pp. 135-137, Mar. 2004.

[20] Rongfang Song and Shu-Huang Leung, "A novel OFDM receiver with second order polynomial Nyquist window function," IEEE Communications Letters, vol. 9, pp. 391-393, 2005.

[21] H.-A. M. Mourad, "Reducing ICI in OFDM systems using a proposed pulse shape," Wireless Personal Communications, vol. 40, pp. 41-48, Jan 2007.

[22] H. F. Arrano and C. A. Azurdia, "ICI reduction in OFDM systems using a new family of Nyquist-I pulses," IEEE Latin America Transactions, vol. 13, no. 11, pp. 3556-3561, 2015.

[23] A. F. Molisch, M. Toeltsch, and S. Vermani, "Iterative methods for cancellation of intercarrier interference in OFDM systems," IEEE Transactions on Vehicular Technology, vol. 56, no. 4, pp. 2158-2167, 2007.

[24] Wen-Sheng Hou and Bor-Sen Chen, "ICI cancellation for OFDM communication systems in time-varying multipath fading channels," IEEE Transactions on Wireless Communications, vol. 4, no. 5, pp. 2100-2110, 2005.

[25] S. Wang, J. S. Thompson, and P. M. Grant, "Closed-form expressions for ICI/ISI in filtered OFDM systems for asynchronous 5G uplink," IEEE Transactions on Communications, vol. 65, no. 11, pp. 4886-4898, 2017.

[26] R. Datta, G. Fettweis, Z. Kollr, and P. Horvth, "FBMC and GFDM interference cancellation schemes for flexible digital radio PHY design," in Euromicro Conference on Digital System Design, pp. 335-339, 2011.

[27] S. Aggarwal, "A survey-cum-tutorial on approximations to Gaussian $Q$ function for symbol error probability analysis over Nakagami-m fading channels," IEEE Communications Surveys Tutorials, vol. 21, no. 3, pp. 2195-2223, Sep. 2019.

[28] J. R. Barry, D. G. Messerschmitt, and E. A. Lee, Digital Communication Third Edition. Norwell, MA, USA: Kluwer Academic Publishers, 2003.

[29] R. M. Corless, G. H. Gonnet, D. E. G. Hare, D. J. Jeffrey, and D. E. Knuth, "On the Lambert- $\mathcal{W}$ function," Advances in Computational Mathematics, vol. 5, no. 1, pp. 329-359, Dec. 1996.

[30] M. Chiani, D. Dardari, and M. K. Simon, "New exponential bounds and approximations for the computation of error probability in fading channels," IEEE Transactions on Wireless Communications, vol. 2, no. 4 , pp. 840-845, Jul. 2003. 